„This is the peer reviewed version of the following article: Annett Reichhelm, Danny Haubold, Alexander Eychmüller (2017). Ligand Versatility in Supercrystal Formation . Adv. Funct. Materials, Volume 27, Issue 39, October 19, 2017, 1700361, which has been published in final form at DOI:10.1002/adfm.201700361.

This article may be used for non-commercial purposes in accordance with Wiley Terms and Conditions for Self-Archiving."

\title{
Ligand Versatility in Supercrystal Formation
}

Annett Reichhelm, Danny Haubold, Alexander Eychmüller ${ }^{*}$

A. Reichhelm, Dr. D. Haubold, Prof. Dr. A. Eychmüller

Department of Chemistry and Food Chemistry, Technische Universität Dresden, 01062 Dresden, Germany

E-mail: Alexander.Eychmueller@chemie.tu-dresden.de

Keywords: supercrystal formation, ligands, surfactants, superlattices, self-assembly

\begin{abstract}
Supercrystals (SCs) offer the opportunity to integrate nanoparticles into current technologies without losing their unique and designable properties. In the past two decades, a lot of research has been conducted, allowing the synthesis of differently shaped nanoparticles of various materials. Employing those building units, several methods have been developed enabling the preparation of an increasing number of different superstructures. In this review, an overview is given of the large versatility of surfactant molecules used for SC preparation. While SCs with uncharged organic ligands are by far the largest group, the use of charged or uncommon ligands allows the preparation of unique SCs and superlattices. Additionally, the influence of the ligands on the selfassembly and the properties of the resulting SCs is highlighted.
\end{abstract}




\section{Introduction}

One nanometer is extremely small. A way to visualize the size dimension of nanoparticles (NPs) is to try to imagine one thousandth of the diameter of a human hair. Accordingly, single NPs are difficult to handle and the implantation of nanometer sized objects into current technologies is a challenging task. For the integration of nanoparticles into technical devices, more manageable larger superstructures are required. On the other hand, it is crucial to avoid the agglomeration of the primary building units in order to preserve their unique size dependent properties. One way to meet these requirements is the preparation of nanoparticle supercrystals (SCs). Those three dimensional superstructures are formed by the self-assembly of NPs, which act as primary building units similar to atoms in common crystals.

There are many terms for superstructures in the current literature and sometimes they get confused. Accordingly, the meaning of the central terms is shown in the following.

Self-assembly. Whitesides and Grzybowski wrote appositely: "Self-assembly is not a formalized subject and definitions of the term self-assembly seem to be limitlessly elastic. As a result, the term has been overused to the point of cliché.”[1] The term has been used for biological systems ${ }^{[2,3]}$, in nanotechnology ${ }^{[4]}$, polymer science ${ }^{[5,6]}$ and many more research fields. Here, the term self-assembly is used to describe the process of crystallization of NPs into a superlattice (Figure 1).

Superstructure and superlattice. The superlattice is an ordered arrangement of the NPs (primary building units) in a three-dimensional lattice. The resulting object is called superstructure (Figure 1b, c, d).

Supercrystal (SC). Supercrystals, super crystals, supracrystals and colloidal crystals are commonly used terms describing a highly-ordered three-dimensional superlattice of 
nanocrystals with external crystal faces and a defined shape comparable to common atomic crystals. (Figure 1b, c).

Mesocrystal. If the NPs within the superlattice have a preferred relative orientation, the resulting SC exhibits a single crystalline diffraction pattern (Figure 1b). Those SCs are called mesocrystals according to the first definition of Cölfen and Antonietti. ${ }^{[7]}$

The field of SCs and self-assembly has emerged early in nanotechnology research. Since first reliable synthetic routes for monodisperse NPs have been developed, self-assembly has been observed: first on TEM grids as small hexagonally arranged thin films (TFs) and later in first SCs. For the self-assembly, the NP solution is destabilized leading to a homogenous nucleation and the formation of supercrystal seeds, which grow within the solution. The self-assembly can be introduced by dissolving the as-prepared NPs in a suitable solvent and slowly evaporating the solvent. By this simple method, well-defined and highly-ordered TFs and SCs have been achieved early. ${ }^{[8]}$ Since these days, many different materials have been successfully self-assembled into SCs ranging from metals to semiconductors, metal oxides and magnetic materials (an overview can be found in Table 2). Additionally, much research has been done to understand the formation mechanism and control the crystallization, leading to a variety of different SCs. Starting from approximately spherical units, also many differently shaped NPs such as cubes ${ }^{[9,10]}$, octahedrons $^{[10]}$, tetrahedrons ${ }^{[11]}$ and nanoplatelets ${ }^{[12]}$ have been self-assembled into various structures. Those new building units provide the opportunity to explore the selfassembly beyond space filling theories of spherical building blocks. ${ }^{[11,13,14]}$

The self-assembly of NPs is commonly introduced by a slow destabilization of the solution. Many different techniques have evolved within the last twenty years ranging 
from simple evaporation ${ }^{[15]}$ to the more complex two-phase $\mathrm{e}^{[16]}$ or gas-phasedestabilization ${ }^{[17,18]}$ methods and experiments with precisely controllable parameters. There are two processes that can take place during the destabilization of the solution: i) homogeneous or ii) heterogeneous nucleation. The homogeneous nucleation describes the aggregation of NPs into a nucleus that can further grow to a SC in the solution, while the aggregation on the substrate surface (heterogeneous nucleation) leads mostly to the formation of ordered thin films (TFs). ${ }^{[19,20]}$ The arrangement of the NPs and their superlattices has been observed to possess a lot of similarities to the atomic arrangement known from common crystals.

Besides all these studies, one aspect has received only minor interest: the ligand. Ligands play a central role during the NP synthesis granting the stability of the nanoparticle dispersion. In some cases, already one of the precursors is stabilized by the ligand. One example for the stabilization of a precursor ion is the lead oleate complex, which is commonly used in the synthesis of PbS or PbSe NPs. ${ }^{[21]}$ Furthermore, the ligand prevents the NPs from agglomeration during growth, storage and handling. Depending on the synthesis route, different ligand types are used (Figure 2). NPs synthesized in organic media are mostly stabilized with long-chain organic molecules. The most common ones are oleic acid (OA, C18), oleylamine (OAm, C18), trioctylphosphine (TOP, C8), trioctylphosphine oxide (TOPO, C8) and dodecanethiol (DDT, C12). Those ligands can be used for almost every material synthesized in organic media. In solution, these surfactants prevent the agglomeration by steric repulsion of the ligands. In aqueous or polar media, NPs are commonly charge stabilized. Common examples are citric acid, cetyltrimethylammonium bromide (CTAB) and cetyltrimethylammonium chloride 
(CTAC), which are as well long-chain organic molecules, but with a charged end granting solubility in polar media. Here, the stability is provided by electrostatic repulsion.

Due to the diversity of the employed ligands, we classified the supercrystal literature into three categories:

- uncharged organic ligands

- charged organic ligands

- unusual ligands

The first category includes uncharged, long-chain organic ligands, which have been used in the large majority of published studies on supercrystals. In addition to these uncharged ligands, the field of charged organic ligands has emerged consisting of organic molecules with a charged end group. The last category includes all the systems that dramatically differ from the others including polymers and DNA. These categories also represent a development in SC assembly starting from the commonly used surfactants to slightly modified versions to completely different systems, which provide new opportunities to design SCs and their properties.

\section{Uncharged Organic Ligands}

Supercrystals with long-chain organic ligands are by far the largest and best known category. A lot of effort has been put into the investigation of the self-assembly of these nanostructures. An overview of the different materials and NP morphologies which have been self-assembled is shown in Table 2. All mentioned systems in this section contain long-chain uncharged organic molecules as surfactants. There are three main ligands, which are used in the majority of the experiments: OA ${ }^{[22-27]}, \mathrm{OAm}^{[22,28]}$ and $\mathrm{DDT}^{[19,29,30]}$ (overview in Figure 2). OA and OAm have the same carbon chain with 18 carbon atoms 
and a cis double bond at C9. The difference is the head group which is a carboxyl group or an amine group for OA and OAm, respectively. Both ligands are useful to stabilize various different types of NPs. DDT has a slightly shorter chain without double bond with only 12 carbon atoms and a thiol head group. Thus, the overall length of the stretched molecules is about the same at around $1.8 \mathrm{~nm}$. The origin of the use of these ligands for SC preparation is directly connected to the development of organic synthetic routes for monodisperse NPs. In many studies, the as-prepared NPs have been washed and used directly for self-assembly by a slow destabilization of the NP solution. Thus, the longchain organic ligands are by far the best studied system allowing a detailed comparison and investigation of the data shown in the current literature. One important aim of supercrystal research is the investigation of influencing factors of the supercrystal formation. This knowledge is necessary for the understanding of the formation process and also for the directed preparation of supercrystals which are optimally adjusted for the respective application. In this chapter, a selection of such influencing factors is discussed with respect to the ligand influence.

\subsection{NP Softness}

Most NPs consist of an inorganic core and an organic shell that protects the NPs from agglomeration by steric repulsion. The surfactant molecules are bound to the inorganic core forming a hairy, flexible shell. In order to estimate the influence of the ligand shell, Boles and Talapin introduced the softness $(S=L / R)$, which is defined as quotient of the ligand length $(L)$ and the radius of the inorganic core $(R) .{ }^{[14]}$ Considering two differently sized NPs with the same ligand and with core diameters of 1 and $100 \mathrm{~nm}$, the share of the soft organic shell in the total volume of the particles is very different. A high $\mathrm{S}$ value corresponds to very soft materials with a large ligand shell in comparison to the core and 
a small value determines a hard system, which might be considered as hard spheres. The softness has been observed to influence the arrangement of the NPs in superstructures. ${ }^{[14]}$ Comparing the softness of the NPs of different SC studies in the literature, the influence of this parameter on the success of supercrystal formation can be observed. In the following, we illustrate the impact of the softness, while an expanded overview of the SC literature is shown in Table 2.

Extensive studies on the size-dependent self-assembly of Au NPs into SCs have been carried out by Pileni and co-workers including the investigation of different particle sizes, surfactants and solvents. ${ }^{[19,29-31]}$ Those studies are perfect model systems for the investigation of the influence of the softness on the formation of supercrystals. The first set of experiments has been carried out using DDT stabilized Au NPs dissolved in toluene at room temperature and slowly destabilizing the NP solution over several hours by the evaporation of the solvent. All parameters are the same, only the core diameter or the ligand is varied. An overview of all experiments and parameters can be found in Table 1. DDT has an effective length of $1.8 \mathrm{~nm}$ as a single, stretched molecule. The NP core diameter varies between 4.3 and $7.8 \mathrm{~nm}$ and thus the softness varies between 0.83 and 0.46. SCs have only been formed if the softness is smaller than 0.7. A larger softness leads only to thin films, while harder systems form well-defined SCs.

In a further study, Pileni and co-workers investigated the self-assembly of 5 and $7 \mathrm{~nm}$ sized Au NPs with different ligands. DDT (C12), tretradecanethiol (C14) and hexadecanethiol (C16) have been used to vary the ligand length and investigate the selfassembly using different preparation methods. At room temperature, only those systems with a softness of less than 0.7 yielded SCs, while the others formed thin films. From these results it seems that 0.7 is a critical barrier for the formation of supercrystals. 
Another set of experiments has been carried out at $50{ }^{\circ} \mathrm{C}$ instead of room temperature. There, it has been shown that SCs can be formed with a softness of up to $0.81 .{ }^{[30]}$ These experiments indicate that higher temperatures can shift the critical softness to higher values.

\subsection{Temperature Dependence}

By changing the preparation temperature, the behavior of the ligands is changed and thus new possibilities emerge. At this point, it should be noted that most of the data presented in the current literature has been obtained at room temperature. Different temperatures were chosen only in a minor fraction of the experiments, while mostly it does not seem necessary as most of the systems already work well at room temperature by using particles with a softness less than 0.7. Nevertheless, there are a few examples, which are highlighted in the next paragraphs.

One of the earliest examples has been shown by Murray et al. ${ }^{[8]}$ They prepared SCs from 2.0, 3.5 and $4.8 \mathrm{~nm}$ sized QDs capped with TOP and TOPO (ligand length $1.1 \mathrm{~nm}^{[32]}$ ) by dissolving the NPs in octane and octanol at $80{ }^{\circ} \mathrm{C}$ and slowly reducing the pressure. The softness corresponds to $1.10,0.63$ and 0.46 . Considering the high softness of 1.10 , it becomes clear that this high temperature was required to form SCs. Furthermore, it was stated that TFs have been prepared at room temperature using a two phase method. In addition, Murray et al. varied the ligand length for $6.3 \mathrm{~nm}$ sized QDs by using differently sized ligands. Those ligands have been trihexadecylphosphine oxide (C16), trioctylphosphine oxide (C8) and tributylphosphine oxide (C4) allowing the tuning of the softness and the interparticle distance between 0.7 and $1.7 \mathrm{~nm}$. This also represents the first investigation of the influence of the ligands on the SC formation. 
Even though Cordeiro et al. did not observe SCs, but TFs, their study impressively shows the temperature effect on the ligands and thus on the interparticle distance. ${ }^{[33]}$ They chose spherical $(6.8 \mathrm{~nm})$ and cubic $(7.5 \mathrm{~nm}) \mathrm{Ce}_{2} \mathrm{O}$ NPs capped with OA as model systems. By carefully washing, they removed as much surfactant as possible while avoiding an agglomeration. At RT, $269 \mathrm{~K}$ and $178 \mathrm{~K}$, samples have been prepared by the so-called sandwich method. Hereby, a droplet of the NP solution was placed onto a TEM grid and covered by a second grid. This special method allowed increasing the assembly quality and ensured a homogenous cooling. The interparticle distance of single ordered layers of spherical NPs has been reduced from 3.4 (RT) to 3.2 (269 K) and 2.6 nm (178 K) (Figure 3). Multilayers have shown an even further reduction of the distance down to $2.3 \mathrm{~nm}$. In this regard, the multilayers are comparable to SCs as the surrounding of the NP influences the interparticle distance. ${ }^{[14]}$ The values for the face-to-face distance of the cubes were about the same as for the spherical ones with only minor differences. Supported by calculations of the total potential energy, they state that the lower solubility of the ligands at lower temperatures causes the reduction of the effective ligand length leading to the decrease of the interparticle distance. Accordingly, the softness of the NP can be lowered by reducing the self-assembly temperature and thus the effective ligand length, providing the possible chance to favor SC formation. Unfortunately, the presented systems cannot be directly compared to the preparation of SCs as the freezing of the droplet when bathing in liquid nitrogen hinders a homogeneous nucleation in solution. Nevertheless, this study gives an insight into the temperature behavior of the ligand.

\subsection{Influence of the Solvent}

Another way to influence the self-assembly is to change the solvent. ${ }^{[29]}$ Using toluene, hexane, octane and cumene as solvents for $5.8 \mathrm{~nm}$ sized Au NPs stabilized by DDT, the 
softness stays unchanged while the ligand-solvent interactions differ. SCs have only been

obtained in toluene and cumene systems, while the other ones lead only to TFs. ${ }^{[29]}$ This observation has been explained by the interplay of van der Waals forces and solventligand and ligand-ligand interactions. In the case of hexane, the stabilizing DDT molecules interact stronger with the solvent than with each other. Hence, the homogenous nucleation into SC seeds would decrease the interactions. Accordingly, no SCs are formed. In contrast to hexane, the interactions between toluene molecules and surfactants are less strong due to the strong attractive interactions between toluene molecules. While the solvent-ligand interactions are rather low, the combined forces of ligand-ligand and van der Waals interactions are strong enough to initiate the SC formation. ${ }^{[29]}$

\subsection{Summary}

For the investigation of the SC formation, long-chain organic surfactants have been largely used for the stabilization of the initial NP building units. From this group of ligands, OA, OAm and DDT have been used in the majority of investigations due to the commonness of NP syntheses using these surfactants. In order to increase the understanding and control of the formation process, several influencing factors like NP size, ligand, temperature and solvent have been extensively studied. According to the collected literature data, the softness of the NPs plays a crucial role during self-assembly. At room temperature, supercrystal formation has been only observed for NPs with a maximal softness of 0.7 , while higher temperatures are needed for the employment of softer particles. In general, long-chain organic ligands are suitable capping agents for the self-assembly of NPs of different shapes and materials. However, they also allow only a limited variation of the self-assembly. Additionally, the properties of the final SC do not only depend on the inorganic core, they are also strongly influenced by the interparticle 
distance and the organic layer between the single building units. The most prominent example of this strong influence is the electrical conductivity. Au NPs should provide a high electrical conductivity, but using long-chain organic molecules as stabilizers leads to the formation of an insulating layer between the single NPs. Thus, only poor conductivities can be achieved. According to our experience, those SCs are almost completely insulating materials and only very little information is found in the literature about measuring the electrical conductivity of such SCs. One of those few studies has been carried out by Yang et al. using scanning tunneling spectroscopy (STM). ${ }^{[34]}$ Those SCs showed sufficient conductivity to be investigated by STM, but far beyond any value that would be interesting for electrical applications. In the next sections, SCs with different ligands are shown which extend the material and structural range of the superlattices, increase the control over the SC formation and influence the properties of the superstructures.

\section{Charged Organic Ligands}

While hydrophobic organic ligands are used for SC preparations in organic solvents, charged molecules are employed for the stabilization of the NPs in polar solvents, e.g. water. Additionally to the results described in the previous section, SCs have also been prepared from aqueous dispersions broadening the range of available SC structures, morphologies and properties. Due to electrostatic interactions, various non-closest-

packed structures are available, which are uncommon in nonpolar SCs. ${ }^{[35]}$ In addition to the driving forces of the self-assembly of nonpolar NPs, charged ligands lead to a number of attractive and repulsive interactions. While equal charges are useful to stabilize NP dispersions, the combination of oppositely charged NPs has been shown to enable an 
electrostatic self-assembly ${ }^{[35]}$. The employment of acid molecules as ligands allows the control of attraction and repulsion by varying the $\mathrm{pH}$ value as explained below.

Aqueous NP syntheses utilize a variety of charged organic ligands. Common examples are citric acid, functionalized thiols and cetyltrimethylammonium chloride (CTAC, Figure 2). Another route to hydrophilic NPs is their synthesis in organic solvents followed by the exchange of the hydrophobic ligands for charged molecules. Despite the large amount of charged ligands, just a few have been used for the preparation of SCs so far. Most of them consist of a long organic chain, a head group for binding to the NP surface and a charged tail group providing hydrophilicity. In this section, an overview of previous approaches is given, sorted by like-charged, oppositely charged and mixed-charged NPs.

\subsection{Like-charged Nanoparticles}

The positively charged ligand cetyltrimethylammonium chloride (CTAC, see Figure 2) has been employed for SC preparation by the group of Michael Huang. CTAC forms a double layer on the NP surface with charged head groups pointing both to the NP surface and into the solution enabling necessary interactions with the solvent. ${ }^{[36-38]}$ Liao et al. ${ }^{[39]}$ used an aqueous dispersion of CTAC stabilized Au NPs. A droplet of the dispersion was placed in a moist environment leading to the slow evaporation of the solvent. The increasing concentration of the dispersion causes the NPs to aggregate into superstructures. By this drop casting method, SCs with various symmetrical shapes and with diameters of some micrometers have been prepared. A distinct influence of the NP shape on the SC morphology has been observed.

In a following study, Yang et al. ${ }^{[40]}$ used the same method and examined the SC formation process by video recorded optical microscopy. In a continuous process, the first SCs were observed shortly after the beginning while the majority reached an observable size after 
20 minutes. In the following minutes, the accretion of NPs from the dispersion led to a quick growth of the SCs into structures of about $3 \mu \mathrm{m}$. In a second approach, the SC formation has been initiated by a gradual increase of the surfactant concentration. ${ }^{[40]}$ Therefore, an Eppendorf tube was filled with the NP dispersion and a CTAC solution was added on top leading to a slow diffusion of the surfactant into the nanoparticle dispersion (Figure 4). During the next 12 hours, the NPs aggregated forming a black precipitate caused by the increasing surfactant concentration. SEM investigations revealed the formation of SCs with sizes between $<1$ and $4 \mu \mathrm{m}$ and symmetrical shapes. By placing a wafer in the dispersion, the possibility of growing SCs directly onto a substrate by this method was shown. The role of the surfactant for the aggregation was confirmed by adding the NP dispersion into a concentrated solution of CTAC. In agreement with the former assumption, NP aggregates were formed by the increase of the surfactant concentration. Here, the rapid flocculation prevents the development of ordered superstructures.

Both the droplet evaporation method and the surfactant diffusion technique were successfully employed by Chiu et al. ${ }^{[41]}$ for the assembly of Au-Pd-core-shell NPs into SCs. Again, the NP shape was observed to strongly influence the morphology of the SCs. By varying the NP concentration, SCs with diameters from 0.5 to $\sim 2 \mu \mathrm{m}$ were obtained, while decreasing the concentration led to smaller SCs. Also, the preparation temperature and the dispersion-surfactant ratio were found to influence the size of the superstructures. Additionally, Au-Ag core-shell and PbS NPs have been assembled into SCs via the surfactant diffusion method to prove the generality of the approach. CTAB (the bromide form, Figure 2) was also successfully employed. The results of these investigations prove 
the suitability of the positively charged ligand $\mathrm{CTA}^{+}$for the preparation of SCs from aqueous dispersions.

Another ligand used for SC preparation is mercaptosuccinic acid (MSA), whose thiol group binds on the NP surface while the deprotonated carboxyl group stabilizes the dispersed NPs by electrostatic repulsion. By lowering the $\mathrm{pH}$ value, protonation occurs leading to the formation of hydrogen bonds as shown by Wang et al. ${ }^{[42]}$ (Figure 5). Employing this method, faceted Au SCs with diameters up to $16 \mu \mathrm{m}$ have been prepared. ${ }^{[42]}$ Alternative to the direct coupling of two carboxyl groups, water molecules form bridges between two groups by hydrogen bonding. ${ }^{[42,43]}$

\subsection{Oppositely charged Nanoparticles}

Charged ligands are not only suitable for the stabilization of NP dispersions, but also have been used to initiate the SC formation. First, Kolny et al. ${ }^{[44]}$ combined dispersions of CdS NPs stabilized with either 2-(dimethylamino)ethanthiol hydrochloride or 3mercaptopropionic acid, leading to the formation of small aggregates, but supercrystals were not achieved yet. For this purpose, the electrostatic interactions of oppositely charged NPs have been investigated in the group of Grzybowski. The chosen ligand molecules were $\omega$-functionalized alkane thiols mercaptoundecanoic acid (MUA) and N,N,N-trimethyl(11-mercaptoundecyl)ammonium chloride (TMA, Figure 2). The thiol groups of these molecules bind to the NP surface, while the negative (MUA) or positive (TMA) charge stabilizes the particles in the aqueous dispersion. In their first study, Kalsin et al. ${ }^{[35]}$ assembled negatively charged Au NPs (AuMUA) and positively charged Ag NPs (AgTMA) by mixing the separate NP dispersions. The average diameter of both NP species has been almost equal leading to comparable spherical building units with opposite charges. After mixing the dispersions, the formed precipitate has been washed 
with water to remove an excess of ammonia salt and has been dispersed in a mixture of dimethyl sulfoxide and water. Afterwards, SCs were prepared by the slow evaporation of the solvent at $70{ }^{\circ} \mathrm{C}$. This so called electrostatic self-assembly (ESA) led to the formation of symmetrical SCs with a sphalerite (diamond like) structure (Figure 6). The assembly into this non-close-packed structure with four oppositely charged neighbors around each NP is due to electrostatic effects at the nanoscale. The obtained truncated tetrahedral and octahedral SCs are typical for the sphalerite structure. The main advantage of the ESA method is the possibility to orderly assemble NPs of different materials in one superstructure combining the properties of both materials. The experiments of Kalsin et al. represent the first binary SCs and by now there are no other examples shown for the self-assembly of different NPs into a free-standing, three-dimensional superstructure. Thus, these studies impressively show the chance of ligand variation to tune the superlattice and the SC properties.

In a following investigation published by Kalsin et al. ${ }^{[45]}$, a dispersion of charged Au NPs has been titrated with dispersed Ag NPs (and vice versa), while the absorption spectra of the mixtures have been observed. Again, MUA and TMA have been used as ligands. Whereas dispersions of like-charged NPs showed additive absorption spectra of the Au and Ag particles, oppositely charged NPs led to modified spectra indicating the formation of core-shell clusters. In these, the shell is formed by the excess NP species causing a net charge of the clusters. Here, efficient Debye screening leads to the stabilization of the clusters in the dispersion. When the ratio of the particle amounts reaches unity, electroneutral aggregates precipitate, which can be redispersed by the addition of one of the NP types. These results are an important step to the general understanding of the 
interactions of charged NPs, which is crucial for the directed preparation of SCs by the ESA approach.

In order to examine the influencing factors of the self-assembly, Kalsin et al. ${ }^{[46]}$ varied the relative concentration of the oppositely charged NP types, the polydispersity and the $\mathrm{pH}$ value. Therefore, a dispersion of AuMUA was titrated with AgTMA until the precipitation occurred. The product was washed and redispersed in a mixture of dimethyl sulfoxide and water. Afterwards, the desired excess of one NP type was added and the $\mathrm{pH}$ was adjusted. SCs were grown by the slow evaporation of the solvent at $60-65^{\circ} \mathrm{C}$. At low dispersities and $\mathrm{pH}=10$, the largest SCs were obtained from dispersions with equal concentrations of positively and negatively charged NPs. Each excess of one type leads to smaller and less regular SCs. A high excess of AuMUA NPs produces unordered structures of connected crystals or amorphous precipitates supposedly due to hydrogen bonding between protonated MUA molecules $(1 \%$ at $\mathrm{pH}=10)$. Counterintuitively, increasing the dispersity of the Ag NPs from 15 to $35 \%$ was observed to improve the quality of the obtained SCs. Since the SCs are exclusively built of the similar sized larger particles, the smaller ones are suggested ${ }^{[35]}$ to slow the aggregation process by screening the electrostatic interactions between the larger particles. Thereby, ordered self-assembly is promoted while fast flocculation is prevented. Lowering the $\mathrm{pH}$ value has been observed to decrease the quality of the crystals. With an excess of MUA capped NPs, unordered, polycrystalline aggregates developed due to the formation of hydrogen bonds between the largely protonated MUA molecules. In summary, the best SCs were grown from dispersions with equal NP concentrations, deprotonated carboxyl groups due to a high $\mathrm{pH}$ value and additional smaller NPs for electrostatic screening. 
Based on the former results, supercrystals have been prepared by Kowalczyk et al. ${ }^{[47]}$ which are stabilized by additional linker molecules to be insoluble in water. Therefore, TMA- and MUA-capped Au and Ag NPs have been assembled by the ESA method as described above. Because the assembled NPs can easily be redispersed in water, the asprepared SCs have been treated with an acetonitrile solution of the cross-linking agent for several hours. Then, the SCs are stabilized with dithiol linkers which connect the NPs at the SC surface. Afterwards, excess dithiol was removed by washing with acetonitrile and the SCs were suspended in deionized water. These stabilized SCs were subsequently used as cores to prepare core-shell SCs. Therefore, a dispersion of oppositely charged NPs was added to the SCs, which act as crystallization seeds for the assembly of the shells while the thiol coating prevents the dispersion of the SCs. Superstructures with a diameter of up to $2.5 \mu \mathrm{m}$ with a $200-300 \mathrm{~nm}$ shell were prepared. Thus, by employing this method, SCs containing sections of different materials can be obtained. Additionally, the analytical application of dithiol-stabilized SCs has been studied. By employing dithiols with cleavable groups, SCs can be designed to indicate desired cleaving analytes. As an example, the $\mathrm{OH}^{-}$caused hydrolysis of an ester group is shown, where the dissolution of the SC is detected by the increasing coloration of the NP dispersion. Hence, the stabilization of SCs with dithiols is an applicable option for both structural designs and analytical applications.

\subsection{Mixed-charged Nanoparticles}

As shown above, charged NPs can be self-assembled from aqueous dispersions, forming large, faceted SCs. This electrostatic self-assembly usually requires the use of two oppositely charged types of NPs. Recently, Pillai et al. ${ }^{[48]}$ presented a method to employ 
this approach to prepare SCs from one single type of NPs, which are stabilized by both positively and negatively charged ligands (Figure 7).

Similar to the previous studies, the functionalized thiols MUA and TMA were used. By soaking the original DDT stabilized NPs in a solution of MUA and TMA, a ratio of 1.6 to 1 of the ligands at the particle surface has been obtained. Due to the protonation and deprotonation of the carboxyl group of MUA, the $\mathrm{pH}$ value is a crucial parameter of the aggregation of these mixed-charged NPs. In alkaline solutions $(\mathrm{pH}=11)$, the MUA ligands are largely deprotonated and hence negatively charged. This charge is partly compensated by the positively charged TMA ligands, but due to the MUA excess, the NPs are negatively charged and form a stable dispersion due to the electrostatic repulsion of the NPs. On the other hand, in acid solutions $(\mathrm{pH}=4)$ nearly all acid molecules are protonated and uncharged, so that consequently the charge of the TMA molecules causes a positive charge of the NPs. Under these conditions, the dispersion is also stable. At $\mathrm{pH}=6$, the MUA is partly protonated while the remaining negative charges are compensated by the positive TMA molecules. Since the net charge of the NPs is zero, there is no repulsive electrostatic force to stabilize the dispersion. Due to hydrogen bonding between the carboxyl groups, flocculation and precipitation of the NPs occur. For the preparation of SCs, dimethyl sulfoxide has been added to the aqueous NP dispersion and the water has been removed by slow evaporation. The resulting precipitate has been examined by SEM, TEM and small angle X-ray diffractometry in order to find the most favorable parameters. While in alkaline solutions $(\mathrm{pH}=11)$ no aggregation occurred due to the electrostatic repulsion of the negatively charged NPs and the lack of hydrogen bonding, at $\mathrm{pH}=4$ SCs were formed. Here, during the evaporation process, a balance between electrostatic repulsion of the positively charged NPs and hydrogen 
bonding leads to a weak attraction between the particles. Under these conditions, the slow self-assembly of the NPs allows the formation of highly ordered SCs. As shown by this investigation, the self-assembly of mixed-charged NPs is an additional route for the preparation of SCs, while the $\mathrm{pH}$ value plays a crucial role for the stability of the NP dispersion and the result of the self-assembly.

\subsection{Summary}

Charged ligand molecules have been employed for the NP self-assembly from aqueous dispersions leading to SCs with unusual structures and properties. In addition to the driving forces of the formation of nonpolar SCs, charged NPs are influenced by electrostatic interactions which enables the formation of non-closest packed superstructures ${ }^{[35]}$ and thus broaden the range of possible SC structures and morphologies. The use of organic acids as ligands allows influencing the stability of the NP dispersion by varying the $\mathrm{pH}$ value. ${ }^{[42,43,48]}$ Thereby, deprotonated carboxyl groups lead to electrostatic repulsion of the NPs und thus stabilize the dispersion and prevent aggregation. On the other hand, at low $\mathrm{pH}$ values protonated carboxyl groups form hydrogen bonds between the particles and induce flocculation or crystallization. ${ }^{[42,43,48]}$ An interesting feature of the self-assembly of oppositely charged NPs is the ordered aggregation of two different materials ${ }^{[35]}$ through the alternating assembly similar to ionic lattices. This approach allows the directed tailoring of nanostructured materials which is useful for the adjustment of the properties to the respective application. Additionally, the mutual influence of the materials can induce new and unique properties. ${ }^{[45]}$ Altogether, the assembly of charged NPs expands the opportunities of nanoparticle self-assembly and has already produced interesting results. 


\section{Unusual Ligands}

In the previous two sections, it has been shown that uncharged and charged long-chain organic surfactants have been used for most SC preparations. Employing those ligands, the structural diversity is limited. In addition, most surfactants are about the same length of around $1.8 \mathrm{~nm}$ and thus, the interparticle distance has been in the range of 2-4 $\mathrm{nm}$ (see Table 2). Those long-chain organic molecules act as an insulating layer between the single NPs leading to poorly conductive SCs. ${ }^{[34,49-51]}$ Furthermore, the as-prepared SCs have been found to be very soft materials lowering their potential to be integrated into common applications. ${ }^{[52-54]}$

In this section, recent approaches are highlighted that overcome those limitations by using completely different ligands ranging from carbon templates and polymers to DNA and other surfactants leading to very unique superstructures.

\subsection{Templates}

Jiao et al. used long-chain organic capped NPs in order to create a carbon template that could be directly used for synthesizing new SCs without the pre-synthesis of the primary building units (scheme shown in Figure 8a). ${ }^{[55]}$ In the first step, $\mathrm{Fe}_{3} \mathrm{O}_{4}$ NPs with a diameter of $11 \mathrm{~nm}$ and OA as capping agent have been synthesized. In the second step, these NPs have been assembled into the well-known SCs with fcc superlattice and common interparticle spacing resulting from the surfactant (Figure 8b, c). The asprepared SCs have then been annealed in order to carbonize the ligands. Here, the $\mathrm{Fe}_{3} \mathrm{O}_{4}$ NPs serve as catalysts allowing the carbonization at lower temperatures at around $500{ }^{\circ} \mathrm{C}$ while oxidation is avoided by working under an inert atmosphere. During the carbonization, a shrinkage of the SCs occurs leading to dramatically reduced interparticle

distances. Nevertheless, the deformation of the SCs is homogenously preserving the 
superlattice and maintaining the shape of the inorganic cores. The former surfactants, which have been carbonized, now form a mesoporous network around the $\mathrm{Fe}_{3} \mathrm{O}_{4} \mathrm{NPs}$. By etching, the cores have been removed leaving the empty network. The measured pore size is smaller than the diameter of the initial cores leading to the assumption of shrinkage during etching.

In the next step, precursors have been infiltrated into the mesoporous network and heated to activate the material formation. Jiao et al. showed that they can successfully prepare $\mathrm{SnO}_{2}, \mathrm{TiO}_{2}, \mathrm{Ti}_{0.3} \mathrm{Sn}_{0.7} \mathrm{O}_{2}$ and Carbon SCs, which have not been achieved by any conventional self-assembly approaches. Those structures can only be prepared by leaving small channels between the inorganic cores allowing the diffusion of the precursors into the template. These channels also lead to interconnected NPs (see also Figure 8d, e) causing excellent electronic properties. This conductivity cannot be achieved by conventional self-assembly of building units capped with organic ligands. In order to characterize these structures for potential energy storage systems, anode materials from $\mathrm{SnO}_{2}$ SCs for lithium ion batteries have been prepared. The measurement of the electrochemical properties showed a capacity of $640 \mathrm{mAh} / \mathrm{g}$ even after 200 cycles. ${ }^{[55]}$ The group has impressively shown the potential of such templates created by self-assembly extending the material range and also interconnecting the primary building units.

\subsection{DNA-hybrids}

The self-assembly of conventional NPs is mostly limited to certain superlattices resulting in similar SCs with similar morphologies. In order to extend the superlattice diversity, Macfarlane et al. prepared NP-DNA hybrids. ${ }^{[56]}$ In the first step, Au NPs with different radii have been synthesized by a common preparation route. Then, the NPs have been functionalized with single strand DNA. This single strand has one end that attaches via a 
thiol head group to the NP surface. This end is followed by a ten base sequence that is a non-binding spacer that can be changed in order to design the interparticle distance. The rest of the DNA molecule is a so-called sticky end, which sticks to complimentary DNA strands that are also bound at the NP surface (corresponding to i, ii, iii and iv in Figure 9B, respectively).

The self-assembly of the NPs is introduced by mixing those complimentary DNA strands leading to chemically interlinked NPs. In this work, Macfarlane et al. studied the influence of the inorganic core diameter, binary mixtures, shell thickness and multiple sticky end combinations. An impressively large structural diversity is presented ranging from simple fcc, bcc and hcp to more complex binary $\mathrm{CsCl}, \mathrm{AlB}_{2}, \mathrm{Cr}_{3} \mathrm{Si}$ and $\mathrm{Cs}_{6} \mathrm{C}_{60}$ structures (Figure 9). They found that they can influence the interparticle distance, the lattice parameters and the crystallographic symmetry of the superlattice by changing the NP size, shell thickness, composition and sticky ends. In comparison to common selfassemblies including binary systems ${ }^{[57-60]}$, such programmable ligands allow superlattice formation beyond the structural range of common long-chain organics. In this regard, the self-assembly by linkage of the DNA ligands allows the directed SC preparation leading to an extended and programmable range of new SCs.

On the one hand, the designable interparticle distance provides the opportunity to couple and decouple the electronic wave functions, controlling composition, location and distance and thus influencing the optical, mechanical and catalytic properties. On the other hand, creating electrical devices from DNA-SCs is prevented by the insulating organic layer between the NP cores leading to a poor electrical conductivity. 


\subsection{Polymers}

In recent years, polymers have developed as a new NP ligand species. ${ }^{[61-63]}$ First experiments showed that polymer capped NPs can be self-assembled into SCs as well. At a first glance, it is obvious that the organic chain of the polymer ligands is much longer than the chain of the previously discussed long-chain organic ligands. This also means that the ligand shell is much thicker. The ligand length can be much more tuned influencing the distance between the inorganic cores. In this regard, the ligand shell cannot be considered as elongated "hairy" surfactant spreading into the solvent. Depending on the size and solubility, the surfactants form a ligand corona around the inorganic core. Influenced by the polymer-solvent interactions, the polymers assemble close to the NP surface or spread into the solvent. In addition, further functionalities can be added by modified monomers.

Shen et al. ${ }^{[64]}$ used $15.2 \mathrm{~nm}$ sized Au NPs coated with the block copolymer PS $17-b$-PAA 83 $\left(M_{n}=1.8 / 6.0 \mathrm{kDa}\right)$ for the preparation of well-defined SCs with an edge length of several hundred nanometers. These SCs exhibit a fcc superlattice with a interparticle distance of $10 \mathrm{~nm}$. The formation has been investigated at different stages following the SC growth. It has been found that the SC seeds are almost exclusively amorphous. In the later stage, the SCs become crystalline forming a fcc superlattice. Interestingly, some of these SCs exhibit a hollow space in the middle. Shen et al. concluded from their observations that the rearrangement of the NPs is introduced from the outer layer to the middle. Due to the fact that the crystallization stops in the center of the SC, a larger space results leading to the formation of such hollow structures. This approach may allow the preparation of new and unique SCs by directing the formation mechanism in order to achieve hollow SCs or infiltrate them with other materials. 
Another approach to self-assemble NPs capped with polymers has been carried out by Henzie et al. ${ }^{[65]}$ They assembled PVP-capped $\left(M_{n}=55 \mathrm{k}\right.$ Da) Ag NP cubes (122 nm), truncated cubes $(68 \mathrm{~nm})$, cuboctahedra $(145 \mathrm{~nm})$, truncated octahedra $(106 \mathrm{~nm})$ and octahedra (300 nm). The self-assembly was carried out in an elongated PDMS chamber allowing the direct tracking of the assembly procedure. In contrast to the former studies, the used NPs are much larger, but also have very bulky PVP ligands with a large molar mass. The interparticle distance has been found to be around $20 \mathrm{~nm}$ and corresponds to the bulky PVP molecules. Even though mostly thin layers and only a few SCs have been formed, this study shows impressively the variety of superlattices that can be achieved by self-assembling NPs with different shapes. A detailed description of the self-assembly of NPs with different shapes can be either found in reference ${ }^{[65]}$ or in other reviews ${ }^{[10,39]}$.

\subsection{Summary}

Even though these are only a few examples for SCs from NPs with unique surfactants, it becomes clear that thereby the opportunity to influence the superlattice and the SC properties is enormous. Interconnected SCs can be achieved by using templating methods, allowing an effective charge carrier transport. Furthermore, the structural range can be dramatically extended by the directed self-assembly via functional surfactants, such as DNA. Also polymers can be used and tuned allowing the SC formation. In addition, the polymers can be functionalized in order to add desired properties to the SC that might be of interest for specific applications. 


\section{Conclusion}

The self-assembly of NPs into well-defined and facetted SCs has been investigated for several years. SCs from various materials and differently shaped NPs have been prepared and new methods have been developed allowing their reliable preparation. In this article, an overview has been given of the different ligands used in supercrystal formation, which have been classified into uncharged, charged and unusual ligands. SCs with uncharged ligands are by far the largest category employing most commonly the three molecules OA, OAm and DDT. Well-defined SCs can be prepared of numerous metals, metal oxides and semi-conductors. Using common uncharged organic ligands, mostly fcc superlattices and a small variety of SC morphologies are observed. It has been found that the softness of the NPs is a crucial parameter that allows a preparation success estimation under given parameters. At room temperature and using a suitable solvent, a softness of 0.7 seems to be a critical value. At higher temperatures, also NP systems with larger softness can be employed. Nevertheless, a large study with a big data set is still missing and would give further evidence to the mentioned findings in this review.

CTAC and CTAB are common charged ligands for NPs allowing the preparation of SCs in polar media. Furthermore, the functionalized thiols mercaptoundecanoic acid (MUA), $N, N, N$-trimethyl(11-mercaptoundecyl)ammonium chloride (TMA) and mercaptosuccinic acid (MSA) have been successfully used for the preparation of SCs. Hereby, the SC formation is influenced by the electrostatic forces between the like-charged or oppositely charged NP surfaces. This also allows the preparation of ordered binary SCs, which has not been achieved by conventional methods.

The last category includes DNA-hybrids, polymer surfactants and templates, which extend the structural versatility enormously. Only a few examples are presented in the 
literature so far. Nevertheless, these show impressively what can be achieved by tuning the ligands of the NPs. Designed superlattices and advanced functional SCs with unique properties can be obtained.

Those examples demonstrate the chances to tune the SC lattice and properties dramatically by the NP surfactants. More research needs to be done on other surfactants than the common uncharged organic ligands. Consequently, it becomes clear that finding new ligands for self-assembling NPs into SCs and adding further properties and features to the superstructures is a key step in nanotechnology. By this, the integration of SCs into current technologies comes closer.

\section{Acknowledgements}

This work was financially supported by the Deutsche Forschungsgemeinschaft through the research training group NANO- AND BIOTECHNOLOGIES FOR PACKAGING OF ELECTRONIC SYSTEMS DFG 1401/2 and the financial support from the European Research Council (ERC-2013-AdG AEROCAT). AR and DH contributed equally to this work.

Received: ((will be filled in by the editorial staff))

Revised: ((will be filled in by the editorial staff)) Published online: ((will be filled in by the editorial staff))

\section{References}

[1] G. M. Whitesides, B. A. Grzybowski, Science 2002, 295, 2418.

[2] S. Zhang, D. M. Marini, W. Hwang, S. Santoso, Curr. Opin. Chem. Biol. 2002, 6, 865.

[3] S. Zhang, Nat. Biotechnol. 2003, 21, 1171.

[4] M. A. Boles, M. Engel, D. V. Talapin, Chem. Rev. 2016, 116, 11220. 
[5] O. Ikkala, G. ten Brinke, Science 2002, 295, 2407.

[6] S. Park, J.-H. Lim, S.-W. Chung, C. A. Mirkin, Science 2004, 303, 348.

[7] H. Cölfen, M. Antonietti, Angew. Chem. Int. Ed. 2005, 44, 5576.

[8] C. B. Murray, C. R. Kagan, M. G. Bawendi, Science 1995, 270, 1335.

[9] H. Chan, A. Demortie, C. E. T. Al, ACS Nano 2012, 6, 4203.

[10] M. H. Huang, S. Thoka, Nano Today 2015, 10, 81.

[11] M. A. Boles, D. V. Talapin, J. Am. Chem. Soc. 2014, 136, 5868.

[12] W. Du, X. Qian, M. Xiaodong, Q. Gong, H. Cao, J. Yin, Chem. - A Eur. J. 2007, $13,3241$.

[13] R. Li, J. Zhang, R. Tan, F. Gerdes, Z. Luo, H. Xu, J. A. Hollingsworth, C. Klinke, O. Chen, Z. Wang, Nano Lett. 2016, 16, 2792.

[14] M. A. Boles, D. V. Talapin, J. Am. Chem. Soc. 2015, 137, 4494.

[15] N. Goubet, J. Richardi, P.-A. Albouy, M.-P. Pileni, J. Phys. Chem. B 2013, 117, 4510.

[16] D. V. Talapin, E. V Shevchenko, A. Kornowski, N. Gaponik, M. Haase, A. L. Rogach, H. Weller, Adv. Mater. 2001, 13, 1868.

[17] P. Simon, E. Rosseeva, I. A. Baburin, L. Liebscher, S. G. Hickey, R. Cardoso-Gil, A. Eychmüller, R. Kniep, W. Carrillo-Cabrera, Angew. Chem. Int. Ed. 2012, 51, 10776.

[18] L. Bahrig, Self-assembly and Mesocrystal Formation via Non-classical Crystallisation, Physical Chemistry, TU Dresden, 2014.

[19] N. Goubet, J. Richardi, P.-A. Albouy, M.-P. Pileni, J. Phys. Chem. Lett. 2011, 2, 417.

[20] M.-P. Pileni, J. Mater. Chem. 2011, 21, 16748. 
[21] S. J. Oh, N. E. Berry, J. H. Choi, E. A. Gaulding, H. Lin, T. Paik, B. T. Diroll, S. Muramoto, C. B. Murray, C. R. Kagan, Nano Lett. 2014, 14, 1559.

[22] L. Bahrig, D. Haubold, F. Röder, S. G. Hickey, A. Eychmüller, MRS Proc. 2014, 1705, 1.

[23] S. M. Rupich, E. V Shevchenko, M. I. Bodnarchuk, B. Lee, D. V. Talapin, JACS 2010, 132, 289.

[24] S. Disch, E. Wetterskog, P. Hermann, G. Salazar-Alvarez, P. Busch, T. Brückel, L. Bergström, S. Kamali, Nano Lett. 2011, 11, 1651.

[25] E. Wetterskog, M. Agthe, A. Mayence, J. Grins, D. Wang, S. Rana, A. Ahniyaz, G. Salazar-Alvarez, L. Bergström, Sci. Technol. Adv. Mater. 2014, 15, 55010.

[26] E. Wetterskog, A. Klapper, S. Disch, E. Josten, R. P. Hermann, U. Rücker, T. Brückel, L. Bergström, G. Salazar-Alvarez, Nanoscale 2016, 8, 15571.

[27] M. Agthe, T. S. Plivelic, A. Labrador, L. Bergström, G. Salazar-Alvarez, Nano Lett. 2016, 16, 6838.

[28] D. Haubold, A. Reichhelm, A. Weiz, L. Borchardt, C. Ziegler, L. Bahrig, S. Kaskel, M. Ruck, A. Eychmüller, Adv. Funct. Mater. 2016, 26, 4890.

[29] N. Goubet, J. Richardi, P.-A. Albouy, M.-P. Pileni, Adv. Funct. Mater. 2011, 21, 2693.

[30] Y. F. Wan, N. Goubet, P.-A. Albouy, M.-P. Pileni, Langmuir 2013, 29, 7456.

[31] N. Goubet, H. Portalès, C. Yan, I. Arfaoui, P.-A. Albouy, A. Mermet, M.-P. Pileni, J. Am. Chem. Soc. 2012, 134, 3714.

[32] B.-H. Kang, S.-W. Lee, S.-W. Lim, J.-S. Kim, G. Sai-Anand, S.-H. Lee, D.-H. Kwon, S.-W. Kang, J. Nanosci. Nanotechnol. 2015, 15, 7169.

[33] M. A. L. Cordeiro, E. R. Leite, E. A. Stach, Langmuir 2016, 32, 11606-11614. 
[34] P. Yang, I. Arfaoui, T. Cren, N. Goubet, M.-P. Pileni, Phys. Rev. B 2012, 86, 1.

[35] A. M. Kalsin, M. Fialkowski, M. Paszewski, S. K. Smoukov, K. J. M. Bishop, B. A. Grzybowski, Science 2006, 312, 420.

[36] F. Bai, D. Wang, Z. Huo, W. Chen, L. Liu, X. Liang, C. Chen, X. Wang, Q. Peng, Y. Li, Angew. Chem. Int. Ed. 2007, 46, 6650.

[37] J. Pérez-Juste, I. Pastoriza-Santos, L. Liz-Marzán, P. Mulvaney, Coord. Chem. Rev. 2005, 249, 1870.

[38] Z. Xu, Y. Hou, S. Sun, J. Am. Chem. Soc. 2007, 129, 8698.

[39] C.-W. Liao, Y.-S. Lin, K. Chanda, Y.-F. Song, M. H. Huang, J. Am. Chem. Soc. 2013, 135, 2684.

[40] C.-W. Yang, C.-Y. Chiu, M. H. Huang, Chem. Mater. 2014, 26, 4882.

[41] C. Y. Chiu, C. K. Chen, C. W. Chang, U. S. Jeng, C. S. Tan, C. W. Yang, L. J. Chen, T. J. Yen, M. H. Huang, J. Am. Chem. Soc. 2015, 137, 2265.

[42] S. Wang, S. Sato, K. Kimura, Chem. Mater. 2003, 15, 2445.

[43] S. Wang, H. Yao, S. Sato, K. Kimura, J. Am. Chem. Soc. 2004, 126, 7438.

[44] J. Kolny, A. Kornowski, H. Weller, Nano Lett. 2002, 2, 361.

[45] A. M. Kalsin, A. O. Pinchuk, S. K. Smoukov, M. Paszewski, G. C. Schatz, B. A. Grzybowski, Nano Lett. 2006, 6, 1896.

[46] A. M. Kalsin, B. A. Grzybowski, Nano Lett. 2007, 7, 1018.

[47] B. Kowalczyk, D. A. Walker, S. Soh, B. A. Grzybowski, Angew. Chem. Int. Ed. 2010, 49, 5737.

[48] P. P. Pillai, B. Kowalczyk, B. A. Grzybowski, Nanoscale 2016, 8, 157.

[49] N. Fishelson, I. Shkrob, O. Lev, J. Gun, A. D. Modestov, Langmuir 2001, 17, 403.

[50] J. B. Pełka, M. Brust, P. Gierłowski, W. Paszkowicz, N. Schell, Appl. Phys. Lett. 
2006, 89, 63110 .

[51] Y. Joseph, I. Besnard, M. Rosenberger, B. Guse, H.-G. Nothofer, J. M. Wessels, U. Wild, A. Knop-Gericke, D. Su, R. Schlögel, A. Yasuda, Tobias Vossmeyer, J. Phys. Chem. B 2003, 107, 7406.

[52] P. Podsiadlo, G. V. Krylova, B. Lee, K. Critchley, D. J. Gosztola, D. V. Talapin, P. D. Ashby, E. V Shevchenko, J. Am. Chem. Soc. 2010, 132, 8953.

[53] C. Yan, I. Arfaoui, N. Goubet, M.-P. Pileni, Adv. Funct. Mater. 2013, 23, 2315.

[54] M. Gauvin, N. Yang, E. Barthel, I. Arfaoui, J. Yang, P. A. Albouy, M.-P. Pileni, J. Phys. Chem. B 2015, 119, 7483.

[55] Y. Jiao, D. Han, Y. Ding, X. Zhang, G. Guo, J. Hu, D. Yang, A. Dong, Nat. Commun. 2015, 6, 6420.

[56] R. J. Macfarlane, B. Lee, M. R. Jones, N. Harris, G. C. Schatz, C. A. Mirkin, Science 2011, 334, 204.

[57] W. Shi, H. Zeng, Y. Sahoo, T. Y. Ohulchanskyy, Y. Ding, Z. Wang, M. Swihart, P. Prasad, Nano Lett. 2006, 6, 875.

[58] X. Ye, J. Chen, B. T. Diroll, C. B. Murray, Nano Lett. 2013, 13, 1291.

[59] D. V. Talapin, E. V Shevchenko, M. I. Bodnarchuk, X. Ye, J. Chen, C. B. Murray, Nature 2009, 461, 964.

[60] J. Wei, N. Schaeffer, M.-P. Pileni, J. Am. Chem. Soc. 2015, 137, 14773.

[61] A. C. Balazs, T. Emrick, T. P. Russell, Science 2006, 314, 1107.

[62] K. S. Soppimath, T. M. Aminabhavi, A. R. Kulkarni, W. E. Rudzinski, J. Control. Release 2001, 70, 1.

[63] B. R. Saunders, M. L. Turner, Adv. Colloid Interface Sci. 2008, 138, 1.

[64] X. Shen, C. Mei, H. He, M. Zhou, W. Xia, X. Zeng, CrystEngComm 2015, 17, 
4637.

[65] J. Henzie, M. Grünwald, A. Widmer-Cooper, P. L. Geissler, P. Yang, Nat. Mater. 2011, 11, 131.

[66] Z. Zhuang, Q. Peng, X. Wang, Y. Li, Angew. Chem. Int. Ed. 2007, 46, 8174.

[67] R. Li, K. Bian, Y. Wang, H. Xu, J. A. Hollingsworth, T. Hanrath, J. Fang, Z. Wang, Nano Lett. 2015, 15, 6254.

[68] K. Soulantica, A. Maisonnat, M. C. Fromen, M. J. Casanove, B. Chaudret, Angew. Chem. Int. Ed. 2003, 42, 1945.

[69] R. Li, K. Bian, T. Hanrath, W. A. Bassett, Z. Wang, J. Am. Chem. Soc. 2014, 136, 12047.

[70] M. Nagel, S. G. Hickey, A. Frömsdorf, A. Kornowski, H. Weller, Z. Phys. Chem. 2007, 221, 427.

[71] Z. Quan, W. S. Loc, C. Lin, Z. Luo, K. Yang, Y. Wang, H. Wang, Z. Wang, J. Fang, Nano Lett. 2012, 12, 4409.

[72] I. Lisiecki, P.-A. Albouy, M.-P. Pileni, Adv. Mater. 2003, 15, 712.

[73] N. Yang, Z. Yang, M. Held, P. Bonville, P.-A. Albouy, R. Lévy, M.-P. Pileni, ACS Nano 2016, 10, 2277.

[74] G. Singh, H. Chan, A. Baskin, E. Gelman, N. Repnin, P. Kral, R. Klajn, Science 2014, 345, 1149.

[75] W. Lu, Q. Liu, Z. Sun, J. He, C. Ezeolu, J. Fang, J. Am. Chem. Soc. 2008, 130, 6983.

[76] A. Pucci, M.-G. Willinger, F. Liu, X. Zeng, V. Rebuttini, G. Clavel, X. Bai, G. Ungar, N. Pinna, ACS Nano 2012, 6, 4382.

[77] I. Kriegel, J. Rodríguez-Fernández, E. Da Como, A. A. Lutich, J. M. Szeifert, J. 
Feldmann, Chem. Mater. 2011, 23, 1830.

[78] Z. Zhao, J. Zhang, F. Dong, B. Yang, J. Colloid Interface Sci. 2011, 359, 351.

[79] M. D. Bentzon, J. van Wonterghem, S. Mørup, A. Thölén, C. J. W. Koch, Philos.

Mag. Part B 1989, 60, 169.

[80] X. Ling, S. Roland, M.-P. Pileni, Chem. Mater. 2015, 27, 414.
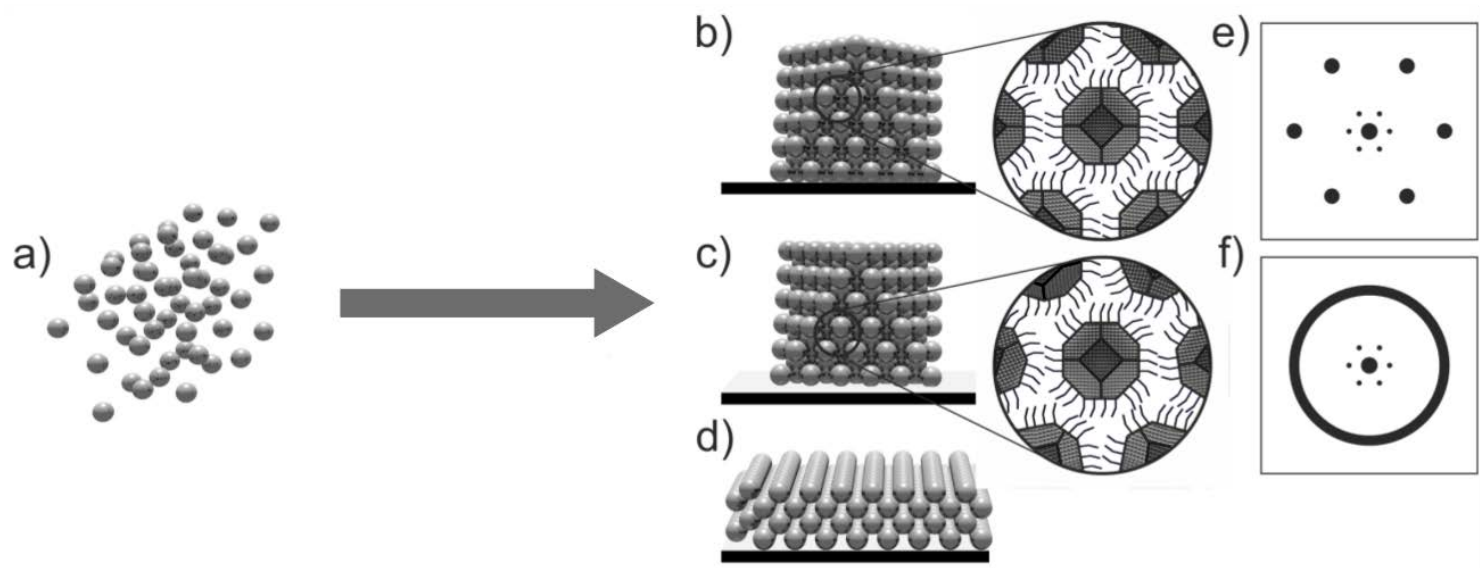

Figure 1. Self-assembly overview. a) NPs from the solution self-assemble into b) mesocrystals, c) SCs or d) thin films (TFs). The magnifications show the NP arrangement in the SCs. e) The NPs in a mesocrystal have a preferred relative orientation which leads to a superposition of the XRD reflections of the atomic crystal (wide angle) and the superlattice (small angle). $\mathrm{f}$ ) If there is no preferred relative orientation, the wide angle reflections overlap to a ring, while the small angle reflections are still spots according to the superlattice. Also TFs can exhibit either single-crystallinity or powder characteristics. 


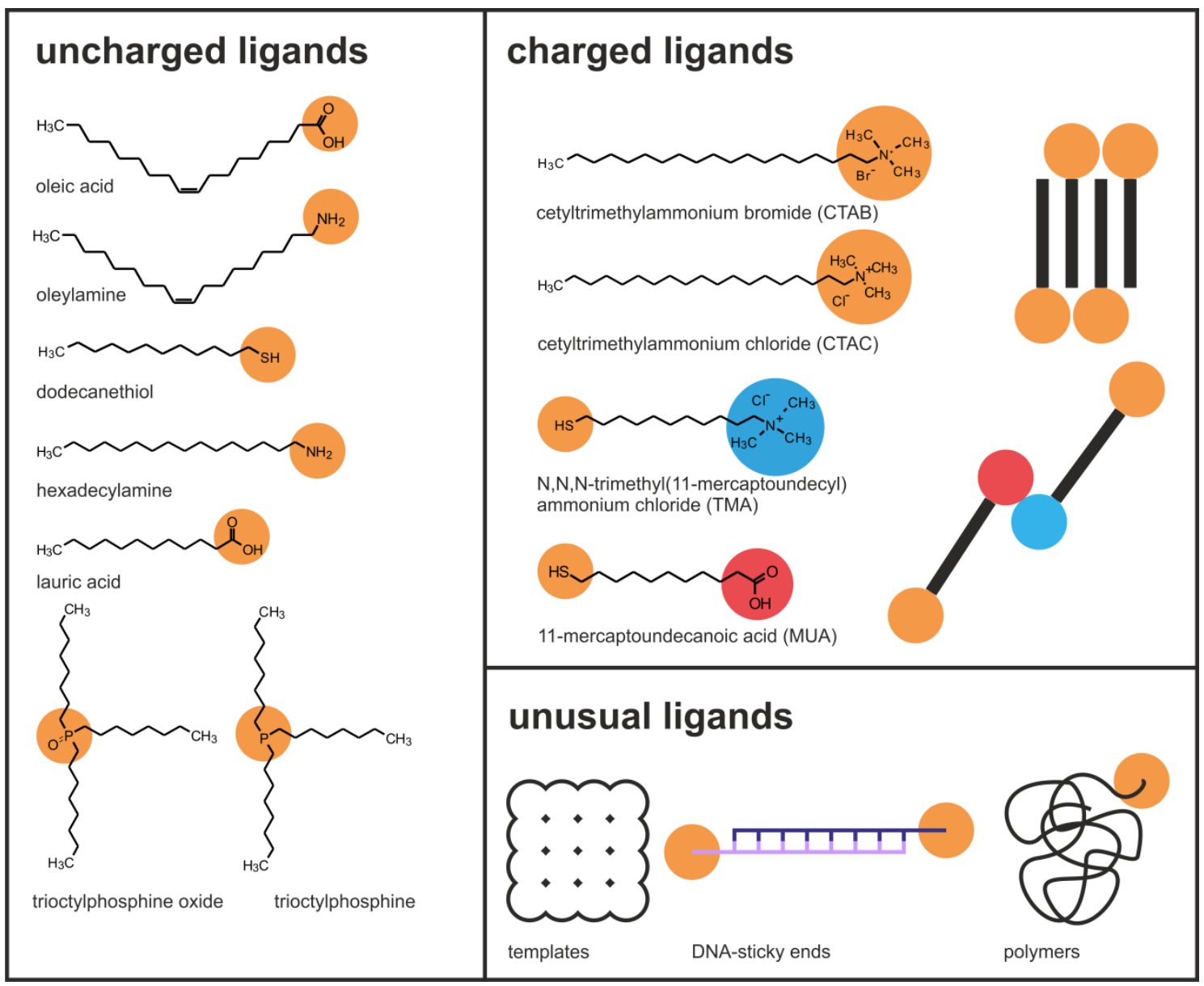

Figure 2. Overview of the common ligands used for stabilizing the NPs during selfassembly into SCs. The ligands are categorized by their stabilization mechanism and functionality. Three main groups are presented in this review: uncharged and charged and unusual ligands. The orange circle marks the functional group that binds to the NP surface, blue and red circles represent charges. The ball-stick scheme illustrate the selfassembly of the corresponding ligands. 

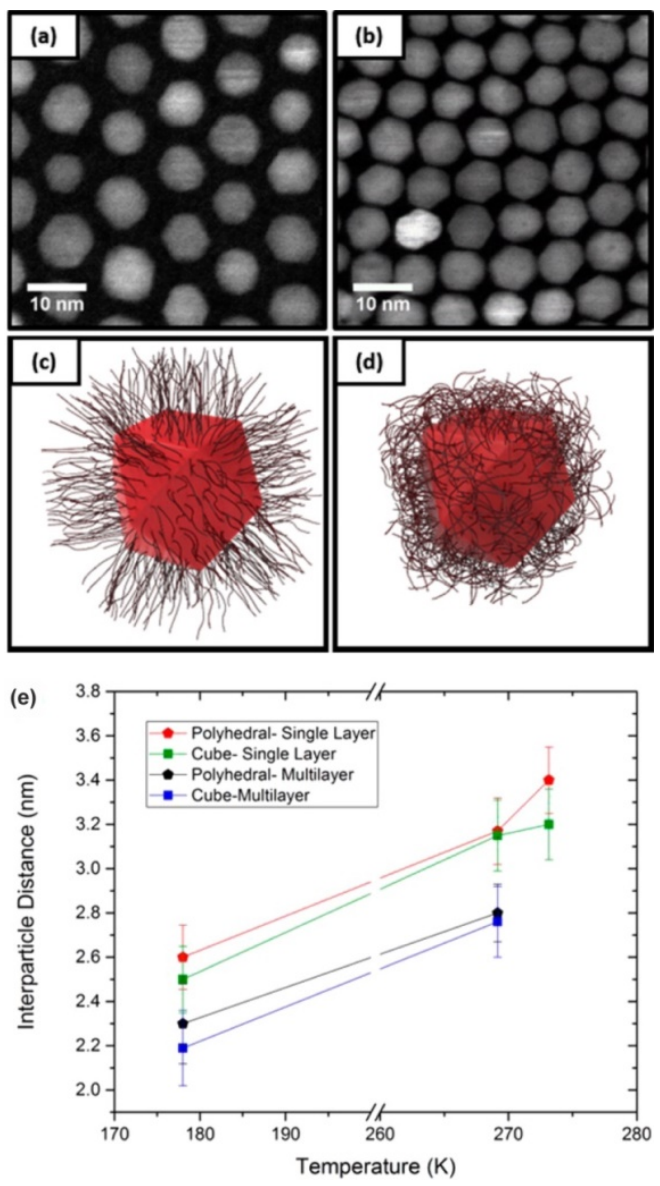

Figure 3. Polyhedral $\mathrm{CeO}_{2}$ NPs prepared at (a) RT and (b) 178 K. (c, d) illustrations of ligand behavior at different temperatures. (e) Interparticle distance (face-to-face) in the superstructures by temperature according to NP shape (polyhedral and cubic) for single and multilayer arrangements. Adapted with permission from Ref ${ }^{[33]}$. Copyright (2006) American Chemical Society.
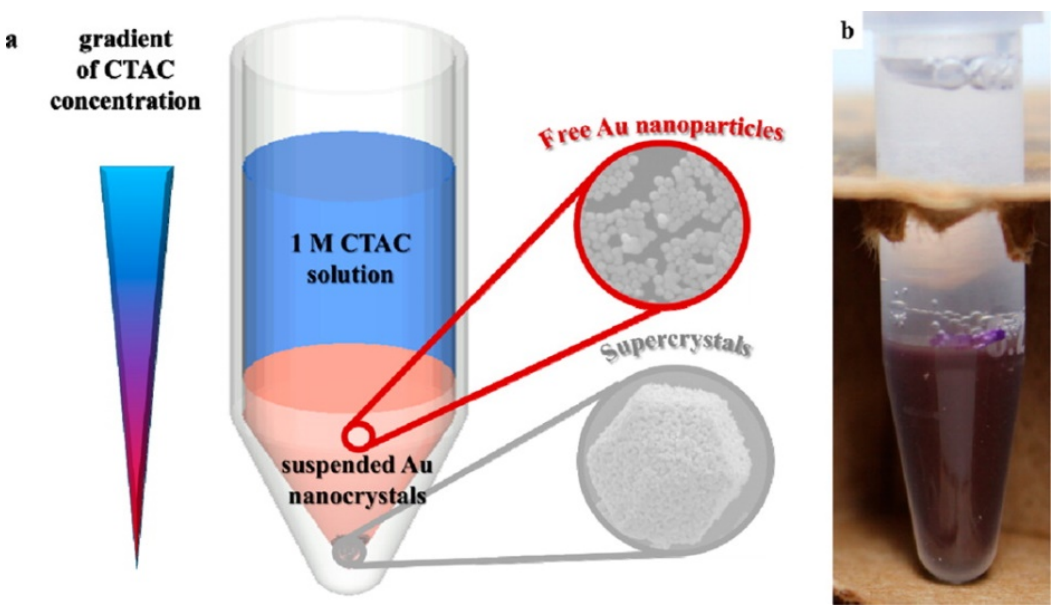
Figure 4. a) Schematic illustration of the surfactant diffusion method where CTAC diffuses from the upper layer into the lower NP solution leading to the formation of SCs. b) Photograph of the vial containing the NP solution and the upper layer of concentrated CTAC solution. Reprint with permission from Ref ${ }^{[40]}$. Copyright (2014) American Chemical Society.

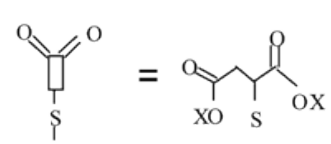

(A)

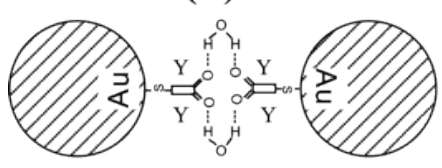

(C)

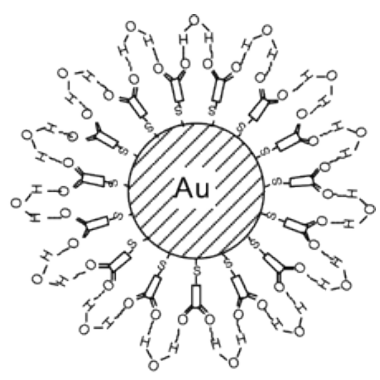

(B)

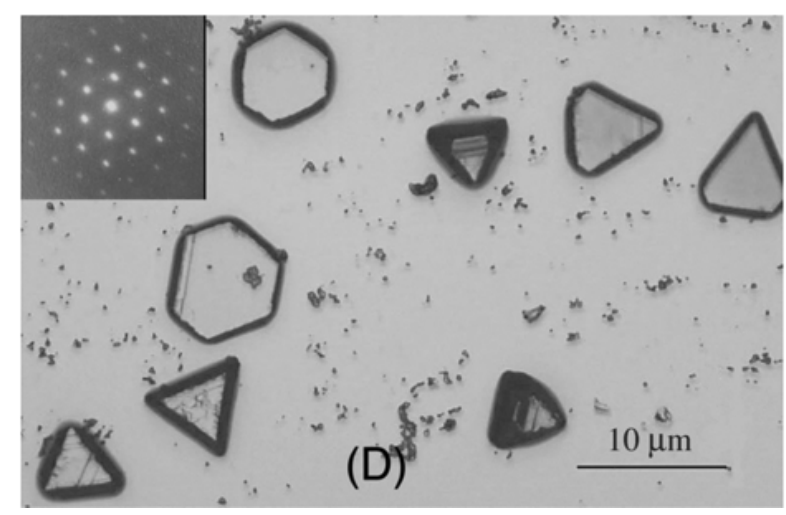

Figure 5. A) Molecular structure of MSA (mercaptosuccinic acid) in which $X$ denotes $H$ in the protonated and Na in the deprotonated form. B) MSA on the surface of an Au NP. Water molecules connect the ligand molecules by hydrogen bonding. C) Water molecules bridging two NPs and hence inducing aggregation. D) Microscope images of Au SCs and low-angle electron diffraction from the superlattice. Reprint with permission from Ref ${ }^{[43]}$. Copyright (2004) American Chemical Society. 

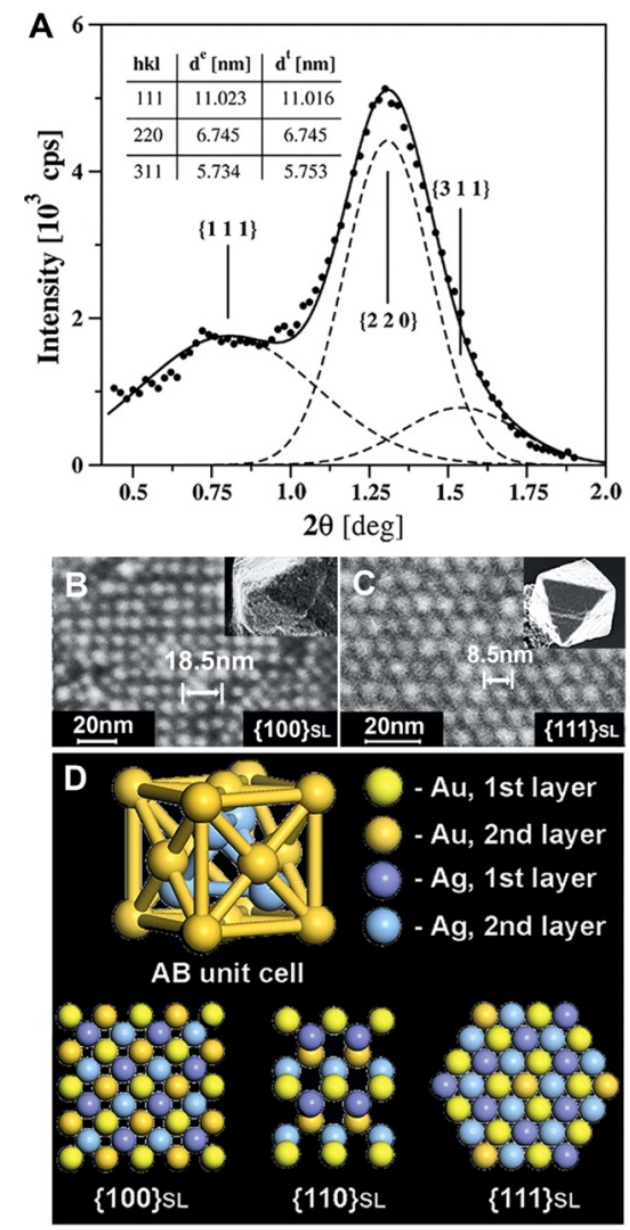

Figure 6. Structure of AuMUA-AgTMA binary crystals. (A) Small-angle powder XRD spectrum of the crystals. Bragg reflections on planes specified by Miller indices shown are characteristic of a diamond-like structure. (Inset) Comparison between experimental $\left(\mathrm{d}^{\mathrm{e}}\right)$ and theoretical $\left(\mathrm{d}^{\mathrm{t}}\right)$ spacing between crystal planes with Miller indices $\{\mathrm{hkl}\}$. Values of $\mathrm{d}^{\mathrm{t}}$ were calculated based on the lattice constant $\mathrm{a}=19.08 \mathrm{~nm}$. The center-to-center distance between NPs on the (100) face, calculated as Embedded Image, is D = 13.49 \pm $0.37 \mathrm{~nm}$; interparticle distance along body-diagonal axis calculated from XRD data is $8.27 \pm 0.26 \mathrm{~nm}$. (B) An SEM image of a \{100\}SL square face taken from a twinnedoctahedron crystal (inset); estimated lattice constant a $=18.5 \mathrm{~nm}$. (C) An SEM image of a $\{111\}$ SL plane of a triangular face of an octahedron (inset) with estimated interparticle distance of $8.5 \mathrm{~nm}$. (D) Scheme of an AB unit cell and the projections of $\{100\} \mathrm{SL}$, $\{110\}$ SL, $\{111\}$ SL planes. NPs of one type are positioned in the nodes of a face-centered cubic lattice, whereas the others occupy half of the tetrahedral voids. The crystals are isostructural with sphalerite ZnS (SG 216) or, for crystals made of only one type of metal cores, with the diamond lattice (SG 227). Reprint with permission from Ref ${ }^{[35]}$. Copyright (2006) AAAS Science. 

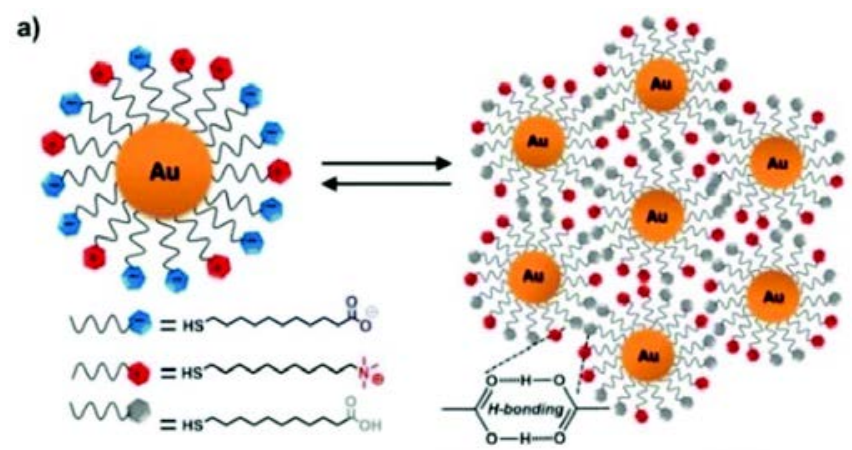

b)

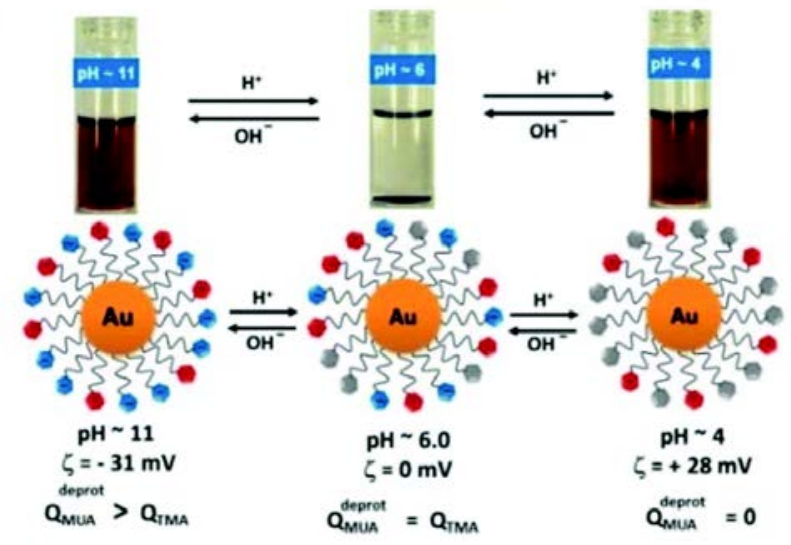

Figure 7. (a) Scheme of NPs stabilized by the oppositely charged ligands MUA and TMA. Under acid conditions, the carboxyl groups are protonated, so hydrogen bonding enables crystal formation. (b) Due to the presence of both acidic and basic groups, the $\mathrm{NPs}$ form stable dispersions at low and high $\mathrm{pH}$ values. When the acid groups are partly protonated at $\mathrm{pH} \sim 6$, the net charge of the NPs is zero and flocculation occurs. Reprint with permission from Ref ${ }^{[48]}$. Copyright (2006) ROYAL SOCIETY OF CHEMISTRY. 

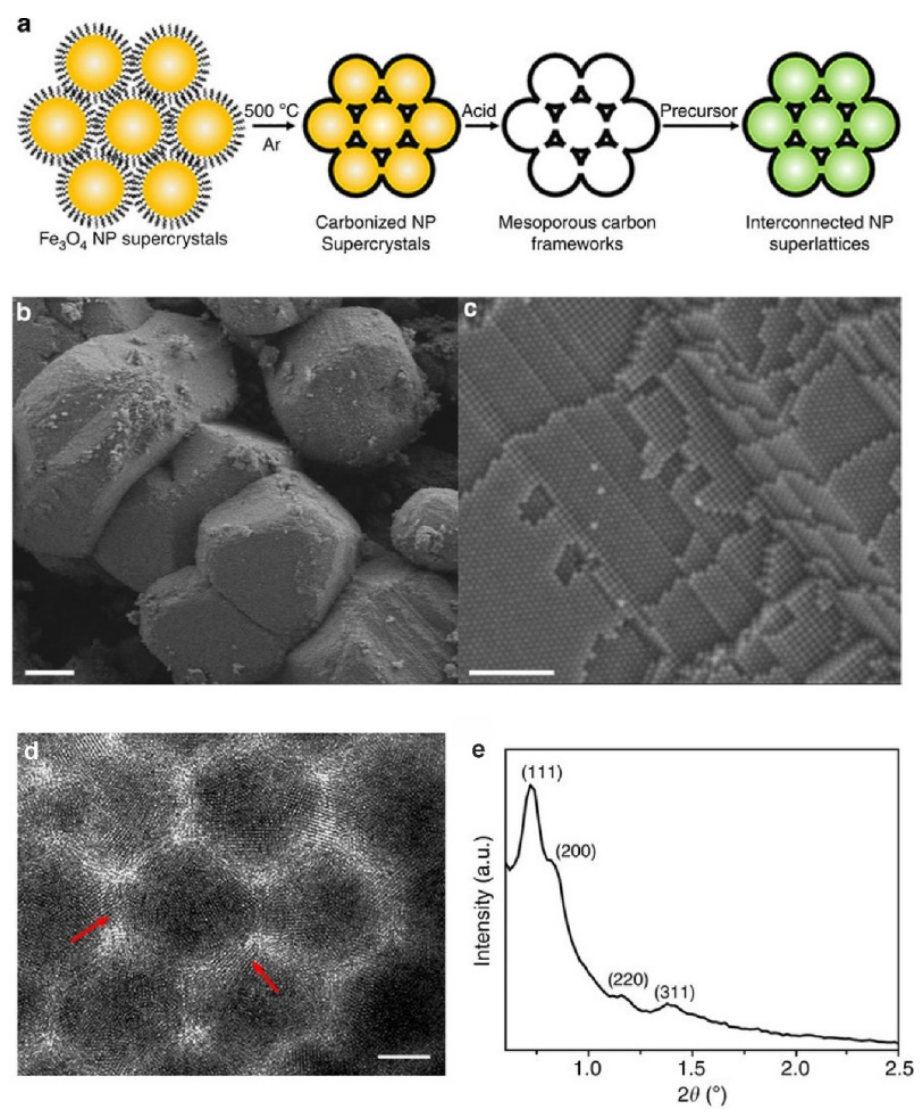

Figure 8. Fabrication of three-dimensionally interconnected NP superlattices from mesoporous carbon frameworks. a) Schematic illustration of the fabrication procedure (cross-sectional view). b, c) SEM and HRSEM images of carbonized $\mathrm{Fe}_{3} \mathrm{O}_{4}$ NP SCs, respectively. Scale bars, $1 \mathrm{~mm}$ and $200 \mathrm{~nm}$, respectively. d) Structural characterization of $\mathrm{SnO}_{2}$ NP superlattices. HRTEM image of $\mathrm{SnO}_{2} \mathrm{NP}$ superlattices showing the high crystallinity of the embedded $\mathrm{SnO}_{2} \mathrm{NPs}$. Scale bar $5 \mathrm{~nm}$. The red arrows indicate NP interconnections. e) SAXS pattern of $\mathrm{SnO}_{2} \mathrm{NP}$ superlattices, respectively. Adapted from ${ }^{[55]}$ 

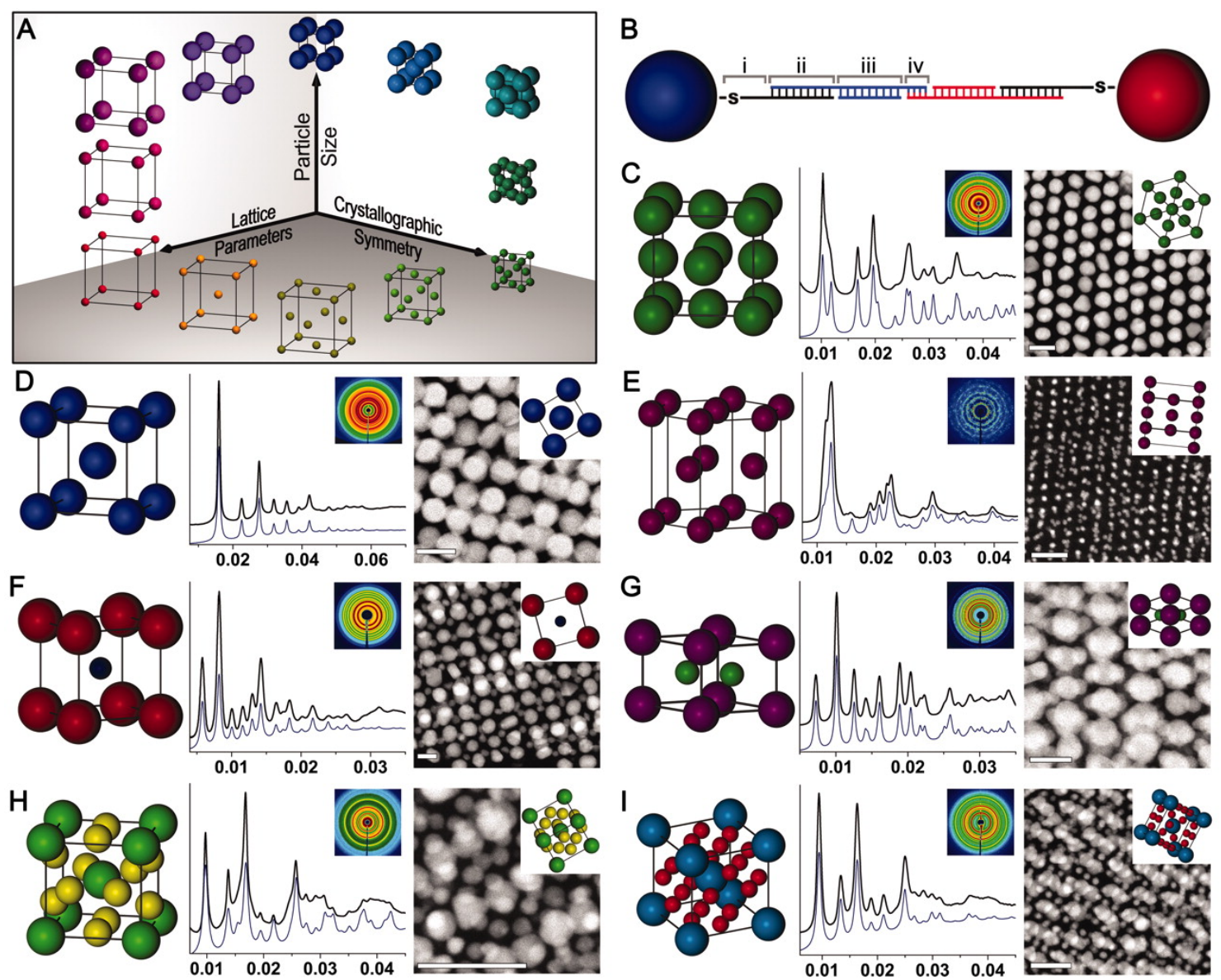

Figure 9. (A) Unlike conventional particle crystallization, NP superlattice engineering with DNA allows the independent control of three important design parameters (particle size, lattice parameters, and crystallographic symmetry) by separating the identity of the particle from the variables that control its assembly. (B) The DNA strands that assemble these NP superlattices consist of (i) an alkyl-thiol moiety and 10-base non-binding region, (ii) a recognition sequence that binds to a DNA linker, (iii) a spacer sequence of programmable length to control interparticle distances, and (iv) a "sticky end" sequence that drives nanoparticle assembly via DNA hybridization interactions. Although only a single linkage is shown schematically here, DNA-NPs typically contain tens to hundreds of DNA linkers per particle. (C to I) The superlattices reported herein are isostructural with (C) fcc, (D) bcc, (E) hcp, (F) CsCl, (G) $\mathrm{AlB}_{2}$, (H) $\mathrm{Cr}_{3} \mathrm{Si}$ and (I) $\mathrm{Cs}_{6} \mathrm{C}_{60}$ lattices. From left to right, each panel contains a model unit cell (not to scale), 1D and 2D (inset) SAXS patterns, and a TEM image of resin-embedded superlattices, along with the unit cell viewed along the appropriate projection axis (inset). Lines in the model denote edges of the unit cell; individual DNA connections are omitted for clarity. SAXS data are plots of NP superlattice structure factor $S(q)$ (y axis, arbitrary units) versus scattering vector q (x axis, $\AA^{-1}$ ). Black traces are experimental data; blue traces are modeled SAXS patterns for perfect lattices. All scale bars in the TEM images are $50 \mathrm{~nm}$. Reprint with permission from Ref ${ }^{[56]}$. Copyright (2011) AAAS SCIENCE. 
Table 1. Dataset from the literature investigating the self-assembly of Au NPs into SCs and thin films (TFs) by discrimination of the NP softness. The table notes the material, ligand, NP radius $R$, interparticle distance $d$, ligand length $L$, superstructure type (SC or $\mathrm{TF}$ ), the solvent, the NP softness $S$ and the corresponding reference.

\begin{tabular}{|c|c|c|c|c|c|c|c|c|c|}
\hline & ligand & $R[\mathrm{~nm}]$ & $d[\mathrm{~nm}]$ & $L[\mathrm{~nm}]$ & SC/TF & SL & solvent & $s$ & Ref \\
\hline \multicolumn{10}{|l|}{ RT } \\
\hline $\mathrm{Au}$ & DDT & 3.9 & 2.0 & 1.78 & $\mathrm{SC}$ & $\mathrm{fcc}$ & toluene & 0.46 & {$[19,29]$} \\
\hline $\mathrm{Au}$ & DDT & 3.9 & 2.5 & 1.78 & $\mathrm{SC}$ & fcc & toluene & 0.46 & [31] \\
\hline $\mathrm{Au}$ & DDT & 3.6 & 2.1 & 1.78 & SC & fcc & toluene & 0.49 & {$[19,29]$} \\
\hline $\mathrm{Au}$ & DDT & 3.5 & 2.6 & 1.78 & SC & $\mathrm{fcc}$ & toluene & 0.51 & [31] \\
\hline $\mathrm{Au}$ & DDT & 3.5 & & 1.78 & SC & $\mathrm{fcc}$ & toluene & 0.51 & [30] \\
\hline $\mathrm{Au}$ & DDT & 3.05 & 2.4 & 1.78 & SC & fcc & toluene & 0.58 & [31] \\
\hline $\mathrm{Au}$ & DDT & 2.9 & 1.9 & 1.78 & SC & fcc & toluene & 0.61 & {$[19,29]$} \\
\hline $\mathrm{Au}$ & DDT & 2.6 & 2.1 & 1.78 & SC & fcc & toluene & 0.68 & [31] \\
\hline $\mathrm{Au}$ & DDT & 2.55 & 1.8 & 1.78 & $\mathrm{TF}$ & fcc & toluene & 0.70 & {$[19,29]$} \\
\hline $\mathrm{Au}$ & DDT & 2.15 & 2.0 & 1.78 & TF & $\mathrm{fcc}$ & toluene & 0.83 & {$[19,29]$} \\
\hline $\mathrm{Au}$ & DDT & 2.9 & 1.9 & 1.78 & $\mathrm{SC}$ & fcc & toluene & 0.61 & [29] \\
\hline $\mathrm{Au}$ & DDT & 2.9 & 1.9 & 1.78 & $\mathrm{TF}$ & fcc & hexane & 0.61 & [29] \\
\hline $\mathrm{Au}$ & DDT & 2.9 & 1.9 & 1.78 & TF & fcc & octane & 0.61 & [29] \\
\hline $\mathrm{Au}$ & DDT & 2.9 & 1.9 & 1.78 & SC & fcc & cumene & 0.61 & [29] \\
\hline $\mathrm{Au}$ & DDT & 2.5 & & 1.77 & TF & & toluene & 0.71 & [30] \\
\hline $\mathrm{Au}$ & tetradecanethiol & 2.5 & & 2.03 & TF & & toluene & 0.81 & [30] \\
\hline $\mathrm{Au}$ & hexadecanethiol & 2.5 & & 2.28 & TF & & toluene & 0.91 & [30] \\
\hline $\mathrm{Au}$ & DDT & 3.5 & & 1.77 & $\mathrm{SC}$ & & toluene & 0.51 & [30] \\
\hline $\mathrm{Au}$ & tetradecanethiol & 3.5 & & 2.03 & SC & & toluene & 0.58 & [30] \\
\hline $\mathrm{Au}$ & hexadecanethiol & 3.5 & & 2.28 & $\mathrm{SC}$ & & toluene & 0.65 & [30] \\
\hline \multicolumn{10}{|l|}{$50{ }^{\circ} \mathrm{C}$} \\
\hline $\mathrm{Au}$ & DDT & 2.5 & & 1.77 & $\mathrm{SC}$ & & toluene & 0.71 & {$[30]$} \\
\hline $\mathrm{Au}$ & tetradecanethiol & 2.5 & & 2.03 & SC & & toluene & 0.81 & [30] \\
\hline $\mathrm{Au}$ & hexadecanethiol & 2.5 & & 2.28 & $\mathrm{TF}$ & & toluene & 0.91 & [30] \\
\hline $\mathrm{Au}$ & DDT & 3.5 & & 1.77 & $\mathrm{SC}$ & & toluene & 0.51 & [30] \\
\hline $\mathrm{Au}$ & tetradecanethiol & 3.5 & & 2.03 & $\mathrm{SC}$ & & toluene & 0.58 & [30] \\
\hline $\mathrm{Au}$ & hexadecanethiol & 3.5 & & 2.28 & $\mathrm{SC}$ & & toluene & 0.65 & {$[30]$} \\
\hline
\end{tabular}


Table 2. Dataset from the literature investigating the self-assembly of NPs into SCs and TFs by discrimination of the NP softness. The table notes the material, ligand, morphology, NP radius $R$, interparticle distance $d$, ligand length $L$, type of superstructure: SC or TL, NP softness $S$ and the corresponding reference the data has taken from.

\begin{tabular}{|c|c|c|c|c|c|c|c|c|c|}
\hline NP & ligand & Morphology & $R[\mathrm{~nm}]$ & $d[\mathrm{~nm}]$ & $L[\mathrm{~nm}]$ & SC/TF & SL & $S=L I R$ & Ref. \\
\hline \multicolumn{10}{|c|}{ uncharged organic ligands } \\
\hline $\mathrm{Au}$ & DDT & spherical & 3.9 & 2.0 & 1.78 & SC & fcc & 0.456 & [29] \\
\hline $\mathrm{Au}$ & DDT & spherical & 3.6 & 2.1 & 1.78 & SC & $\mathrm{fcc}$ & 0.494 & [29] \\
\hline $\mathrm{Au}$ & DDT & spherical & 2.9 & 1.9 & 1.78 & SC & fcc & 0.614 & [29] \\
\hline $\mathrm{Au}$ & DDT & spherical & 2.55 & 1.8 & 1.78 & TF & fcc & 0.698 & [29] \\
\hline $\mathrm{Au}$ & DDT & spherical & 2.15 & 2.0 & 1.78 & TF & fcc & 0.828 & [29] \\
\hline $\mathrm{Au}$ & DDT & spherical & 2.9 & 1.9 & 1.78 & TF & fcc & 0.614 & [29] \\
\hline $\mathrm{Au}$ & DDT & spherical & 2.9 & 1.9 & 1.78 & TF & fcc & 0.614 & [29] \\
\hline $\mathrm{Au}$ & DDT & spherical & 2.9 & 1.9 & 1.78 & SC & fcc & 0.614 & [29] \\
\hline $\mathrm{Au}$ & DDT & spherical & 2.15 & 2.0 & 1.78 & TF & fcc & 0.828 & [19] \\
\hline $\mathrm{Au}$ & DDT & spherical & 2.55 & 1.8 & 1.78 & TF & fcc & 0.698 & [19] \\
\hline $\mathrm{Au}$ & DDT & spherical & 2.9 & 2.0 & 1.78 & SC & fcc & 0.614 & {$[19]$} \\
\hline $\mathrm{Au}$ & DDT & spherical & 3.55 & 2.2 & 1.78 & SC & fcc & 0.501 & [19] \\
\hline $\mathrm{Au}$ & DDT & spherical & 2.6 & 2.1 & 1.78 & SC & fcc & 0.685 & [31] \\
\hline $\mathrm{Au}$ & DDT & spherical & 3.05 & 2.4 & 1.78 & SC & fcc & 0.584 & [31] \\
\hline $\mathrm{Au}$ & DDT & spherical & 3.5 & 2.6 & 1.78 & SC & fcc & 0.509 & [31] \\
\hline $\mathrm{Au}$ & DDT & spherical & 3.9 & 2.5 & 1.78 & SC & fcc & 0.456 & [31] \\
\hline $\mathrm{Au}$ & CTAC & various & $18.5-21.5$ & & 1.6 & SC & various & $0.086-0.074$ & [39] \\
\hline $\mathrm{Au}$ & CTAC & octahedral & $19.5-21.5$ & & 1.6 & SC & & $0.082-0.074$ & [40] \\
\hline $\mathrm{Au}$ & CTAC & rhombic & $26-31.5$ & & 1.6 & SC & & $0.062-0.051$ & [40] \\
\hline $\mathrm{Au}$ & mercaptosuccinic acid & spherical & 1.85 & 1.4 & 0.75 & SC & hсp & 0.405 & [42] \\
\hline $\mathrm{Au}$ & mercaptosuccinic acid & spherical & & & & & hcp & 0.405 & [42] \\
\hline $\mathrm{Au}$ & DDT & spherical & 3.5 & & 1.78 & SC & fcc & 0.509 & {$[30]$} \\
\hline $\mathrm{Au}$ & tetradecanethiol & spherical & 3.5 & & & SC & fcc & & [30] \\
\hline $\mathrm{Au}$ & hexadecanethiol & spherical & 3.5 & & & SC & fcc & & [30] \\
\hline $\mathrm{Ag}$ & OAm & spherical & 2.75 & & 1.8 & SC & fcc & 0.655 & [28] \\
\hline $\mathrm{Ag}$ & OAm & spherical & 4.4 & & 1.8 & TF & fcc & 0.409 & {$[28]$} \\
\hline $\mathrm{Ag}$ & OAm & spherical & 5.65 & & 1.8 & TF & fcc & 0.319 & [28] \\
\hline $\mathrm{Ag}$ & OAm & spherical & 6.25 & & 1.8 & TF & fcc & 0.288 & [28] \\
\hline $\mathrm{Ag}_{2} \mathrm{~S}$ & DDT & spherical & 1.75 & & 1.8 & SC & fcc & 1.028571429 & [66] \\
\hline $\mathrm{Pt}$ & $\mathrm{OA}$ and $\mathrm{OAm}$ & cubic & 4.75 & 2.8 & 1.8 & SC & $\mathrm{R}-3 \mathrm{~m}$ & 0.379 & [67] \\
\hline $\mathrm{Pt}_{3} \mathrm{Ni}$ & $\mathrm{OA}$ and $\mathrm{OAm}$ & octahedral & 4.63 & $1.0 \& 4.64^{\star}$ & 1.8 & & bcc & 0.389 & [13] \\
\hline $\mathrm{Sn}$ & $\mathrm{HDA}^{*} \mathrm{HCl}$ & mixed & & & & SC & hcp & 0.389 & [68] \\
\hline $\mathrm{PbS}$ & OA/TOP & spherical & 2.75 & & $1.8 / 1.1$ & SC & fcc & $0.655-0.400$ & {$[17]$} \\
\hline $\mathrm{PbS}$ & OA & spherical & 5.65 & & 1.8 & SC & fcc & 0.319 & [69] \\
\hline $\mathrm{PbS}$ & TOPO/OA & spherical & 5 & $0.3(3.2)$ & $1.8 / 1.1$ & SC & fcc & $0.36-0.22$ & [70] \\
\hline $\mathrm{PbS}$ & OA & cubes & 6.5 & $2.6^{\star}$ & 1.8 & SC & tilted fcc & 0.277 & [71] \\
\hline Co & lauric acid & spherical & 3.6 & & 1.77 & TF & & 0.492 & [72] \\
\hline Co & OA & spherical & 4.1 & 2.8 & 1.8 & & fcc or hcp & 0.439 & [73] \\
\hline
\end{tabular}




\begin{tabular}{|c|c|c|c|c|c|c|c|c|c|}
\hline Co & OA, TOPO & spherical & 3.5 & 2.5 & 1.8 & SC & fcc & 0.514 & [54] \\
\hline Co & OA, TOPO & spherical & 4 & 2.3 & 1.8 & SC & fcc & 0.450 & [54] \\
\hline Co & OA, TOPO & spherical & 4.5 & 2.7 & 1.8 & SC & fcc & 0.400 & [54] \\
\hline $\mathrm{Fe} 3 \mathrm{O} 4$ & OA & cubic & & & 1.8 & strings & complex & & [74] \\
\hline $\ln 2 \mathrm{O} 3$ & OAm & octahedral & & & & TF/SC & 3 types mixed & & [75] \\
\hline CdSe & TOP/TOPO/HDA & spherical & 1.75 & & 1.1 & SC & $\mathrm{fcc}$ & 0.629 & [16] \\
\hline CdSe & TOP/TOPO & sphercial & 2.4 & 1.1 & $1.1^{*}$ & $\mathrm{SC}$ & fcc & 0.458 & [8] \\
\hline CdSe & trihexadecylphosphate & sphercial & 2.4 & 1.7 & & $\mathrm{SC}$ & fcc & & [8] \\
\hline CdSe & tributylphosphine oxide & sphercial & 2.4 & 0.7 & & SC & fcc & & [8] \\
\hline $\mathrm{ZnO}$ & benzoate & spherical & 1.85 & 4.09 & & SC & fcc or P6/mmm* & & [76] \\
\hline $\mathrm{ZnO}: \mathrm{Tb}$ & benzoate & spherical & 1.5 & & & SC & $\mathrm{P} 6 / \mathrm{mmm}$ or $\mathrm{R}-3 \mathrm{~m}^{*}$ & & [76] \\
\hline ZnO:Y & benzoate & spherical & 2.15 & & & SC & $\mathrm{R}-3 \mathrm{~m}$ & & [76] \\
\hline Cu1.97S & $\begin{array}{l}\text { OA/OAm/ } \\
\text { DDT }\end{array}$ & spherical & 6.3 & & 1.8 & SC & unkown & 0.286 & [77] \\
\hline \multicolumn{10}{|c|}{ larged organic ligands } \\
\hline $\mathrm{Au}, \mathrm{Ag}$ & MUA(Au), TMA(Ag) & spherical & & & $\begin{array}{c}1.63 \mathrm{MUA}, \\
1.9 \mathrm{TMA}\end{array}$ & $\mathrm{SC}$ & sphalerite & $0.320(\mathrm{Au}) ; 0.396(\mathrm{Ag})$ & {$[35,48]$} \\
\hline $\mathrm{Au}, \mathrm{Ag}$ & MUA, TMA & spherical & & & $\begin{array}{c}1.63 \mathrm{MUA}, \\
1.9 \mathrm{TMA}\end{array}$ & $\mathrm{SC}$ & & & [45] \\
\hline $\mathrm{Au}, \mathrm{Ag}$ & MUA(Au), TMA(Ag) & sperical & $\begin{array}{l}\text { Au } 2.6 \\
\text { Ag } 2.7\end{array}$ & & $\begin{array}{c}1.63 \mathrm{MUA}, \\
1.9 \mathrm{TMA}\end{array}$ & SC & & & [46] \\
\hline $\mathrm{Au}, \mathrm{Ag}$ & MUA(Au), TMA(Ag) & sperical & $\begin{array}{l}\text { Au } 2.6 \\
\text { Ag } 2.7\end{array}$ & & $\begin{array}{c}1.63 \mathrm{MUA}, \\
1.9 \mathrm{TMA}\end{array}$ & $\mathrm{SC}$ & & & [48] \\
\hline Au-Pd & CTAB/CTAC & various & $17.50-30.00$ & $3.7 / 6.5$ & 1.6 & SC & different & $0.091-0.053$ & [41] \\
\hline $\mathrm{PbS}$ & CTAB & octahedron & & & & SC & fcc & & [78] \\
\hline $\mathrm{PbS}$ & CTAB & octahedron & & & & $\mathrm{SC}$ & fcc & & [78] \\
\hline $\mathrm{PbS}$ & CTAB & trunc. octahedron & & & & SC & fcc & & [78] \\
\hline \multicolumn{10}{|c|}{ usual ligands } \\
\hline $\mathrm{Fe} 3 \mathrm{O} 4$ & OA & spherical & 5.5 & $5.12^{\star}$ & 1.8 & SC & fcc & 0.327 & [55] \\
\hline C & carbon templates & network & & & & & fcc & & [55] \\
\hline $\mathrm{SnO} 2$ & carbon templates & network & & & & & fcc & & [55] \\
\hline $\mathrm{Fe} 3 \mathrm{O} 4$ & OA & spherical & 3.45 & 2.2 & 1.8 & SC & fcc & 0.522 & [79] \\
\hline $\mathrm{Au}$ & DNA & spherical & & & various & SL & variety & 0.06 & [56] \\
\hline $\mathrm{Ag}$ & PVP & various & & & & $\mathrm{SC} / \mathrm{TF}$ & complex & & [65] \\
\hline $\mathrm{Au}$ & PS-b-PAA & spherical & $50-300$ & 8.6 & & $\mathrm{SC}$ & fcc & & [64] \\
\hline $\mathrm{Au}$ & $\mathrm{N}$-heterocyclic carbenes & spherical & & & various & TF & fcc & & [80] \\
\hline
\end{tabular}




\section{Authors}

Annett Reichhelm received her B.Sc. and M.Sc. degree in chemistry in 2014 and 2016 from Technische Universität Dresden, Germany. In 2016, she started her PhD studies on the selfassembly of semiconductor nanoparticles into supercrystals in the group of Prof. Alexander Eychmüller.

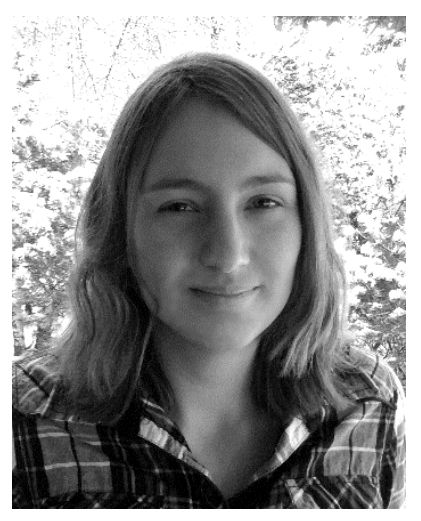

Danny Haubold studied chemistry at the Technische Universität Dresden and received his Bachelor's and Master's degree in 2011 and 2013, respectively. In 2013, he started his PhD studies in the group of Prof. Alexander Eychmüller working on the self-assembly of metal and semiconductor nanoparticles into highly-ordered superstructures. In 2016, he joined the group of Prof. Dmitri V. Talapin for a six months research stay extending his knowledge in self-assembling.

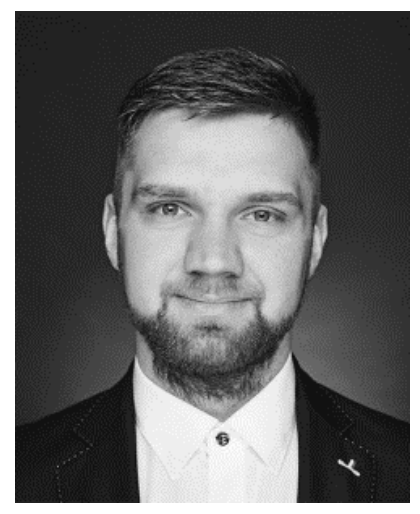

Alexander Eychmüller The academic career of Alexander Eychmüller started in Göttingen with studies of physics (Ph.D. at University of Göttingen and MPI for Biophysical Chemistry, Prof. A. Weller and Dr. K. H. Grellmann) and continued at UCLA (postdoc with Prof. M. A. El-Sayed), Berlin (HMI with Prof. A. Henglein), and the University of Hamburg (with Prof. H. Weller). Since 2005 he holds the chair in Physical Chemistry at TU Dresden. His research interests include the synthesis and characterization of nanosized objects and their photophysical, electrochemical and structural properties. 


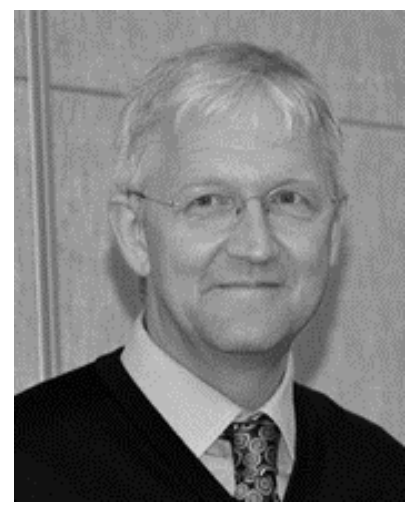

\section{Table of contents}

In this feature article, the influence of the surfactant species on the supercrystal formation is discussed with regard to superlattice and structural diversity. The variety of different ligands is categorized into three groups, which are uncharged long-chain organics, charged organics and unusual ligands. While the first category includes the mostly used ligands, uncommon surfactants enable the preparation of unique superstructures.

Keywords: supercrystal formation, ligands, surfactants, superlattices, self-assembly

Annett Reichhelm" Danny Haubold ${ }^{\#}$, Alexander Eychmüller*

\section{Ligand Versatility in Supercrystal Formation}

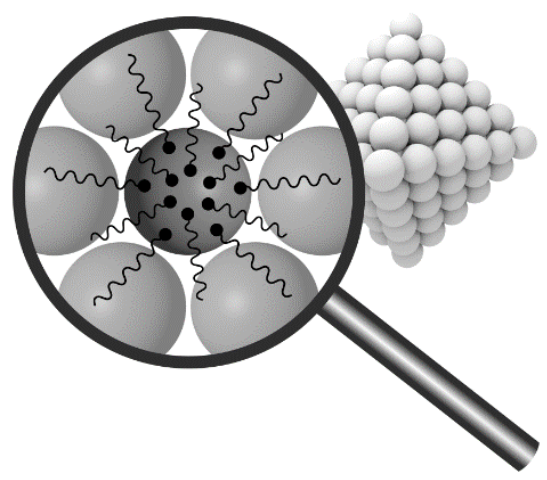

